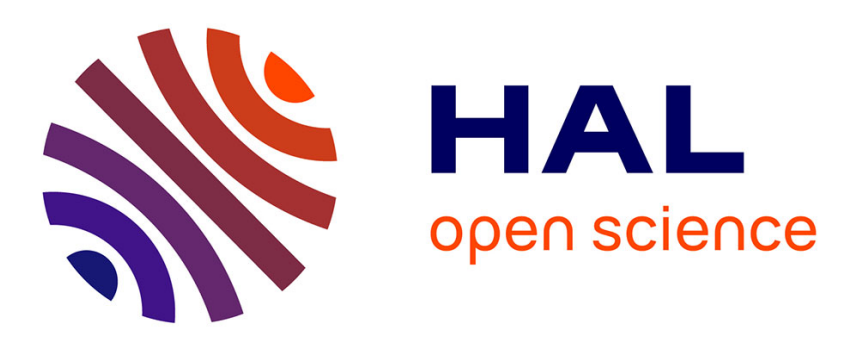

\title{
A comparison between off and on-body control surfaces for the FW-H equation: Application to a non-compact landing gear wheel
}

\author{
Antoine Hajczak, Laurent Sanders, François Vuillot, Philippe Druault
}

\section{To cite this version:}

Antoine Hajczak, Laurent Sanders, François Vuillot, Philippe Druault. A comparison between off and on-body control surfaces for the FW-H equation: Application to a non-compact landing gear wheel. Journal of Sound and Vibration, 2021, 490, pp.115730. 10.1016/j.jsv.2020.115730 . hal-03071714

\author{
HAL Id: hal-03071714 \\ https://hal.science/hal-03071714
}

Submitted on 16 Dec 2020

HAL is a multi-disciplinary open access archive for the deposit and dissemination of scientific research documents, whether they are published or not. The documents may come from teaching and research institutions in France or abroad, or from public or private research centers.
L'archive ouverte pluridisciplinaire HAL, est destinée au dépôt et à la diffusion de documents scientifiques de niveau recherche, publiés ou non, émanant des établissements d'enseignement et de recherche français ou étrangers, des laboratoires publics ou privés. 


\title{
A Comparison Between Off and On-Body Control Surfaces for the FW-H Equation: Application to a Non-Compact Landing Gear Wheel
}

\author{
Antoine Hajczak ${ }^{\mathrm{a}, *}$, Laurent Sanders $^{\mathrm{a}}$, François Vuillot ${ }^{\mathrm{b}}$, Philippe Druault ${ }^{\mathrm{c}}$ \\ ${ }^{a}$ DAAA, ONERA, Université Paris Saclay, F-92322 Châtillon - France \\ ${ }^{b}$ DMPE, ONERA, Université Paris Saclay, F-91123 Palaiseau - France \\ ${ }^{c}$ Sorbonne Université, Institut Jean Le Rond d'Alembert, UMR CNRS 7190, F-75005 Paris - France
}

\begin{abstract}
This work is a contribution to the ongoing debate about the role of quadrupoles in low Mach number flows, studied through the use of solid and permeable surface Ffowcs-Williams \& Hawkings (FW-H) integrals for landing gear numerical noise predictions. It rests upon the key idea that the dominance of surface sources over volume sources can only be guaranteed when the compact-source condition is met. We propose here to express this property as a condition on the smallness of the product between the Mach and Strouhal numbers, or formally as $M S t<1$. We consider a canonical isolated wheel, that basically consists in a shallow circular cavity inscribed in a coin-like cylinder, thus presenting few different length scales, as compared to a full landing gear assembly. Zonal Detached Eddy Simulations are performed on several gradually refined grids to assess the grid convergence of the numerical result. In particular, important computational effort is put into the accurate resolution of acoustic waves up to Strouhal numbers such that $M S t>1$. Overall, determining the real significance of quadrupoles was challenging as numerical errors or misleading effects such as near-field terms, surface discretization, or source domain truncation biased the initial permeable surface results. Even when these sources of bias are removed, non-negligible differences are found between the solid and permeable noise spectra at non-compact $S t$ values, while both formulations are equivalent at lower Strouhal numbers. The analysis of these differences is extended to the determination of their impact on frequency-domain noise maps obtained with the DAMAS algorithm fed with far-field signals computed with both FW-H approaches. A tentative interpretation of the wheel noise sources is finally proposed, highlighting the dominant role of the scattering of aerodynamic sources by the cavity downstream edge at low Strouhal numbers, while wake sources dominate at higher Strouhal numbers.
\end{abstract}

Keywords: Landing Gear, Aeroacoustics, Acoustic Analogy

\section{Introduction}

Early predictions of landing gear noise date back to the end of the 70s, when it was recognized as a dominant airframe noise source. These predictions have initially relied on the use of semi-empirical models, like the ones proposed by Fink [1], Smith \& Chow [2] or Guo [3]. Such models only require a few basic quantities like the number of wheels and their diameter, the length of the main strut, the Mach number, and consequently allow quick noise predictions desirable in the industry. Their main drawback is

${ }^{*}$ Corresponding author

Email address: antoine.hajczak@onera.fr (Antoine Hajczak) 
that they contain an important part of empiricism, which makes their use somewhat limited to the landing gear noise databases they were calibrated with. Ever since the first simulations in the early 2000s (see, eg, Hedges et al. [4], Souliez et al. [5], Lockard et al. [6]), CFD has become an essential tool for landing gear noise predictions. In particular, the progress achieved in turbulence modelling and the increase in computational power have allowed the simulation of the turbulent flow field around landing gears with a degree of complexity ranging from moderate (see among others De la Puente et al. [7]) to almost industrial (see recent LBM-VLES simulations of Bouvy et al. [8]) with very satisfying accuracy.

The vast majority of numerical far-field landing gear noise predictions resort to a so-called hybrid method, which consists in two steps that are (i) the computation of the unsteady turbulent flow in the vicinity of the landing gear and (ii) its extrapolation to far-field acoustic perturbations by means of an acoustic analogy. The most popular in the case of landing gear noise is the Ffowcs-Williams \& Hawkings [9] (FW$\mathrm{H})$ analogy that provides an inhomogeneous wave equation with source terms accounting for the presence of solid surfaces in arbitrary motion. In practice, the formal solution of this equation involves surface and volume integrals of equivalent multipolar source terms on an arbitrarily chosen closed surface and in its outer volume. The volume integral is in most practical situations not manageable. As for the surface terms, two approaches exist, known as the permeable and solid formulation, respectively. The most general is the permeable one and is equivalent to the Kirchhoff method if the surface is taken large enough. This formulation takes into account the quadrupole volume sources enclosed by the surface as well as the interaction between turbulence and the solid surface of the body, of dipole nature. Despite being theoretically exact, this formulation is notoriously cumbersome as the surface has to be located in the linear region to avoid spurious noise generation associated with source domain truncation. The second formulation, the solid one, is the most widely used for landing gear noise predictions. As the surface is taken coincident with the rigid surfaces, it avoids spurious noise associated with the wake and is additionally less computationally expensive as only wall pressure fluctuations need to be saved. These advantages come however with the hypothesis that quadrupoles can be neglected. This still remains a source of debate in the general case, and is the concern of the present work.

A specificity of landing gear noise is that, by definition, it is only relevant at approach conditions, where the Mach number upper bound for a commercial airplane is about 0.25. This argument is often invoked to justify the use of the solid formulation as it is commonly stated that, when $M \ll 1$, dipoles are much more efficient than quadrupoles as their contributions to the mean-squared acoustic pressure scale with $M^{6}$ and $M^{8}$, respectively. These types of scaling laws can however be contested. For instance, Spalart [10] suggested that the scaling law for quadrupoles in the presence of dipoles might be $M^{7}$, rather than $M^{8}$. Moreover, the theoretical $M^{6}$ scaling law is only valid in the compact dipole case, formally expressing the enhancement of the direct quadrupole field radiation by a scattered dipole field, as shown analytically by Davies [11] on the case of multipole diffraction by a rigid sphere, and later illustrated numerically by Gloerfelt et al. [12] on the problem of the aerodynamic noise emitted by a circular cylinder. A surface of dimension $L$ is said acoustically compact whenever the condition $L / \lambda \ll 1$ is fullfilled, $\lambda$ being the acoustic wavelength. This ratio, as pointed out by several researchers [10, 11, 13], can also be formed by the product $M \times S t$, showing that a condition of the type $M S t<1$ might be more relevant than just $M \ll 1$ for neglecting the quadrupole contribution. In the case of a landing gear, the value of $L$ that is relevant to define compactness can be difficult to find, as pointed out by Dobrzynski [14], and might as well be dependant on the listener position. The most natural choice could be the wheel diameter, but Spalart [10] argued that this distance could be greater if, for instance, the landing gear is mounted on a rigid plane. On the grounds of these theoretical considerations, the hierarchy of the multipolar terms, and therefore the use of a formulation instead of the other appears controversial in the scope of landing gear noise predictions, 
especially if high Strouhal numbers are to be considered.

This point of contention is not specific to landing gear noise, and is also illustrated by several airframe noise studies, even for Mach numbers below 0.25. For a circular cylinder in a crossflow, Pérot et al. [15] have found that the FW-H surface integral was dominated by the volume integral (explicitly computed) for the broadband radiation. A similar conclusion was reached by Zhang et al. [16], who used compressible wall-resolved LES. In the tandem cylinders test case, Brès et al. [17] have reported a closer match with experimental data for the highest frequencies when using a permeable surface approach. Greschner et al. [18], and later Giret et al. [19], have pointed out that the quadrupolar sound emitted by a rod airfoil configuration at $M=0.2$ was no longer negligible and that the permeable formulation better matched the experimental results of Jacob et al. [20] at high frequencies for an integration surface surrounding the whole rod-airfoil system and a portion of its wake. Wolf et al. [21], supported by Yu \& Lele [22], have shown that in the case of a wake interaction, quadrupolar noise emission was mainly concentrated in the high frequency range. They found that it could be neglected for a Mach number of 0.1, but not for 0.3 and 0.5 . Souliez et al. [5] and Spalart et al. [23] have claimed a possible importance of near-field quadrupoles with the permeable surface for landing gears at Mach numbers below 0.23. De la Puente [24] has compared the solid and permeable approaches on the Partially Dressed-Cavity Closed (PDCC) test case $(M=0.166)$ and found that, while the solid approach yielded better results with respect to flyover measurements, the permeable approach performed better for sideline listeners thanks to the inclusion of the acoustic reflexions on the fuselage mouting plate. Finally, Appelbaum et al. [25] have recently performed the aeroacoustic simulation of a full scale aircraft with an LBM approach at a Mach number of 0.23 . They have compared both the solid and the permeable approaches to direct noise computation and found a closer match with the permeable integration surface than with the solid surface in the high frequency range.

All the points raised above motivated us to propose a comparison between both the solid and permeable formulations of the FW-H equation in a configuration representative of a landing gear flow. Such comparison has of course already been made, for instance on the LAGOON [26], PDCC [27] or RLG [23] test cases. However, the inherent geometrical complexity of a full landing gear, even stripped from its smallest components, seems to remain too high to address this problem, specially to determine a relevant length scale to discriminate frequencies at which the body is compact. In the recent years, there has been some interest in studying landing gear flows at the isolated subcomponent level, such as the main strut/torque link interaction [28, 29] or facing [30, 31] and tandem wheels [32]. An isolated wheel prototype has been thoroughly studied at the University of Southampton by Wang [33]. As for ONERA's contribution, a first computation has been performed by De la Puente et al. [34] on an isolated wheel from the LAGOON configuration, followed by an in-depth analysis of the circular cavity flow inside the wheel. The computation of the far-field was then restricted to the solid formulation with a relatively short timelength. A subsequent paper was presented by the present authors at the 2018 AIAA/CEAS Aeroacoustics Conference (see Ref. [35]), in which a new grid was designed to accurately resolve the wake quadrupoles and evaluate their contribution with a dedicated permeable integration surface and longer time signals. The comparison between the solid and permeable results systematically showed differences of several $\mathrm{dB}$ in the low frequency range at all angles. For a downstream observer, differences from 5 to $10 \mathrm{~dB}$ were observed at all frequencies, that were attributed to spurious radiation caused by the wake crossing the surface. On the other hand, both formulations gave equal noise levels on large frequency intervals for observers not too close to the flow direction. This very good partial agreement, along with the apparently unphysical behavior at downstream angles, led us to conclude that quadrupoles could be neglected and that remaining differences were only due to numerical errors caused by turbulence crossing the surface.

The present article expands those previous results with some additional analysis. As no experimental 
or numerical reference database are available, new aerodynamic results are presented, grid convergence is assessed in addition to comparisons with similar geometries, supporting the reliability of the simulation. The most downstream observers were rather close to the integration surface, so the effect of near-field terms of the FW-H equation, not addressed in previous results, has been assessed in the present study. The use of corrective terms in the FW-H integral to mitigate spurious noise created by aerodynamic disturbances on the surface downstream part is also presented for the first time. The numerical consistency of the FW$\mathrm{H}$ integration is demonstrated by comparison with Direct Noise Computation (DNC), and verification of nil pressure field inside the FW-H surface. Despite all these points being treated, some differences still remain in noise spectra in the high frequency range and seem inescapable. These differences are supported by the theoretical arguments and work from other researchers listed above, challenging our own former conclusions. In this perspective, an original analysis of the noise sources on the LAGOON wheel is proposed by inputting the far-field signals computed with the solid and permeable approaches to a frequency-domain deconvolution algorithm for the localisation of acoustic sources. Based on the obtained noise maps, an interpretation of the remaining differences in noise spectra is proposed, suggesting a possible ambiguity when, in the presence of non-compact bodies, the sole surface pressure fluctuations are considered as noise sources.

\section{The FW-H equation and its implementation in ONERA's solver KIM}

The process by which the energy carried by a turbulent flow is converted to acoustic perturbations is described by Lighthill's well-known equation. This equation is obtained by rearranging the NavierStokes equations into an inhomogeneous wave equation that can then be solved with the Green's functions formalism. This methodology forms the basis of an acoustic analogy and obviates the high computational cost that would be required to propagate sound waves to the far-field with limited dissipation and dispersion errors. Ffowcs-Williams \& Hawkings [9] have then extended this methodology to account for the presence of surfaces in arbitrary motion in the flow. Let $(\Sigma: f=0)$ be a mathematical surface, not necessarily coincident with the body of interest, such that $f<0$ inside the surface, $f>0$ outside and $\vec{\nabla} f=\vec{n}$ (see figure 11 .

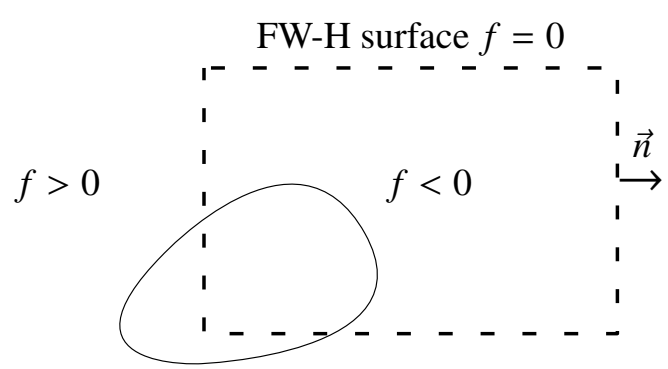

Physical surface

Figure 1: Illustration of the mathematical surface $(f=0)$ used in the FW-H formalism

The surface is moving with respect to the reference frame at a velocity $\vec{v}^{\Sigma}$ and the flow velocity is noted $\vec{u}$. After the developments of Di Francescantonio [36] for a "permeable" surface, the FW-H equation reads as follows:

$$
\square^{2}\left[c_{0}^{2} \rho^{\prime} H(f)\right]=\frac{\partial^{2}}{\partial x_{i} \partial x_{j}}\left[T_{i j} H(f)\right]-\frac{\partial}{\partial x_{i}}\left[L_{i} \delta(f)\right]+\frac{\partial}{\partial t}\left[\rho_{\infty} U_{n} \delta(f)\right]
$$


Where $\delta(f)$ and $H(f)$ stand for the Dirac distribution and Heaviside function, respectively. The different source terms, with the viscous stress tensor denoted $\tau_{i j}$, are:

$$
\begin{array}{r}
P_{i j}^{\prime}=p^{\prime} \delta_{i j}-\tau_{i j} \\
T_{i j}=\rho u_{i} u_{j}+P_{i j}^{\prime}-c_{0}{ }^{2} \rho^{\prime} \delta_{i j} \\
L_{i}=P_{i j}^{\prime} n_{j}+\rho u_{i}\left(u_{j}-v_{j}{ }^{\Sigma}\right) n_{j} \\
U_{n}=u_{j} n_{j}+\left[\left(\rho / \rho_{\infty}\right)-1\right]\left(u_{j}-v_{j}{ }^{\Sigma}\right) n_{j}
\end{array}
$$

The solution can be analytically expressed by convolution with the free-field 3D Green's function and thus reads:

$$
4 \pi c_{0}^{2} \rho^{\prime} H(f)=\frac{\partial^{2}}{\partial x_{i} \partial x_{j}} \int_{V_{(f>0)}} \underbrace{\left[\frac{T_{i j}}{r\left|1-M_{r}\right|}\right]_{\mathrm{ret}}}_{\text {(a) }} \mathrm{d} V-\frac{\partial}{\partial x_{i}} \int_{\Sigma} \underbrace{\left[\frac{L_{i}}{r\left|1-M_{r}\right|}\right]_{\mathrm{ret}}}_{\text {(b) }} \mathrm{d} S+\frac{\partial}{\partial t} \int_{\Sigma} \underbrace{\left[\frac{\rho_{\infty} U_{n}}{r\left|1-M_{r}\right|}\right]_{\mathrm{ret}}}_{\text {(c) }} \mathrm{d} S
$$

where []$_{\text {ret }}$ means that the time-dependent variable inside the brackets is evaluated at the retarded-time $\tau_{\text {ret }}=t-r / c_{0}, r$ being the source-observer distance, and $M_{r}=v_{i}{ }^{\Sigma} r_{i} / c_{\infty}$.

The calculation of the far-field acoustic pressure requires the evaluation of the three integrals described previously, that are respectively known as:

(a): the volumic quadrupolar source term that accounts for the noise directly generated by the turbulence,

(b): the surface loading source term (dipolar) that describes the noise generated by the interaction between turbulent fluctuations and solid boundaries of the body,

(c): the surface thickness term (monopolar) related to the fluid displacement when the body is in motion.

This equation has been derived from the Navier-Stokes equations without any loss of generality. The major drawback is then the computational cost associated with the evaluation of the Lighthill stress tensor $T_{i j}$ in the volume exterior to the integration surface, which is out of reach in most industrially relevant problems. In practice, two approaches are then possible. In both cases, it is common to neglect the stress tensor $\tau_{i j}$ for high Reynolds number flows, giving simply $P_{i j}=p^{\prime} \delta_{i j}$.

- The first, and the most commonly used, is known as the solid formulation of the FW-H equation. The basic idea is to take the surface $\Sigma$ coincident with the solid boundaries of the body considered $\Sigma^{\text {sol }}$ so that only the pressure has to be saved on the rigid walls. If, additionnally, the walls are impermeable and non-vibrant $\left(u_{n}=v_{n}{ }^{\Sigma}\right)$ and the surface is stationary $((c)=0)$, the FW-H equation reduces to:

$$
4 \pi c_{0}^{2} \rho^{\prime} H(f)=\frac{\partial^{2}}{\partial x_{i} \partial x_{j}} \int_{V_{(f>0)}} \frac{\left[T_{i j}\right]_{\mathrm{ret}}}{r} \mathrm{~d} V-\frac{\partial}{\partial x_{i}} \int_{\Sigma^{\mathrm{sol}}} \frac{\left[p^{\prime} n_{j} \delta_{i j}\right]_{\mathrm{ret}}}{r} \mathrm{~d} S
$$

As already mentioned in the introduction, Gloerfelt et al. [12] have numerically illustrated that the surface term of Eq. 8 exactly represents the scattering of aerodynamic sources by the rigid surface in the flow, while the volume term represents the radiation by these same aerodynamic sources if 
the rigid surfaces were not present. Scattering can substantially increase the radiation efficiency of a given source, provided that the corresponding source region is compact [37]. Only in this case, a hierarchy of the multipolar terms is possible and dimensional analysis shows that the dipolar term follows the $M^{6}$ law, while the quadrupolar term follows the $M^{8}$ law. If the latter is neglected, then the gain in computational cost is substantial, as the equation is advantageously reduced to:

$$
4 \pi c_{0}^{2} \rho^{\prime} H(f)=-\frac{\partial}{\partial x_{i}} \int_{\Sigma^{\mathrm{sol}}} \frac{\left[p^{\prime} \delta_{i j}\right]_{\mathrm{ret}}}{r} \mathrm{~d} S
$$

This formulation is then very convenient for industrial applications if the wall pressure involved in the surface integral is accurate enough. In the case of a compact body, retarded-time variations are small and the wall pressure can be described by an incompressible flow model. On the other hand, it has been shown by Schram et al. [38] that in the case of non-compact geometries, using Curle's equation with an incompressible description of the wall pressure perturbations could lead to significant error in the far-field.

To the best of our knowledge, it is still not totally clear to what extent using a compressible solver, which would ideally provide the acoustic component in the wall-pressure fluctuations, allows to relax the compact source argument when using the solid formulation. This particular point was raised by $\mathrm{Yu}$ $\&$ Lele [22], who reported that, even with some of the compressibility effects taken into account in the pressure term thanks to their compressible LES approach, explicit inclusion of the wake quadrupoles was needed to obtain accurate noise predictions.

- The second approach is to design a control surface $\Sigma^{*}$ that contains the body and all the most turbulent regions. The idea is that the Lighthill tensor vanishes outside $\Sigma^{*}$, such that:

$$
\int_{V(f>0)} \frac{\left[T_{i j}\right]_{\text {ret }}}{r\left|1-M_{r}\right|} \mathrm{d} V=0
$$

If $\Sigma^{*}$ is also stationary, the equation reduces to:

$$
4 \pi c_{0}^{2} \rho^{\prime} H(f)=-\frac{\partial}{\partial x_{i}} \int_{\Sigma^{*}} \frac{\left[p^{\prime} \delta_{i j}+\rho u_{i} u_{j}\right]_{\mathrm{ret}} n_{j}}{r} \mathrm{~d} S+\frac{\partial}{\partial t} \int_{\Sigma^{*}} \frac{\left[\rho u_{i} n_{i}\right]_{\mathrm{ret}}}{r} \mathrm{~d} S
$$

thus resulting in a Kirchoff-like integration, provided that the flow leaving the surface on its endcap 150 closure is not too turbulent, so the surface can be considered in the linear region. It has been suggested that a quadrupole crossing the surface end might be chopped into two dipoles that are much more efficient acoustically [18]. The one inside the surface would eventually cause spurious noise generation, not being compensated by the ignored volume integration. In order to mitigate this phenomenon, corrective terms based on the estimation of the Lighthill source term flux across the surface have been derived [39, 40, 41]. Other ideas consist in averaging several outflow surfaces in order to remove the hydrodynamic component from the integration, as proposed by Shur et al. [42], or applying a window function to progressively turn the surface contribution to zero in the streamwise direction.

Equations 1 to 11 have been written in the case of a propagation medium at rest for simplicity reasons. The extension to the case where the propagation medium is a uniform flow is easily obtained and will be adopted in practice in the rest of the article. In the absence of spurious noise, and if the permeable surface is well resolved, the difference between the pressure computed with both formulations is expected to exactly 
represent the quadrupole contribution to the far-field. To this end, the code KIM, developed at ONERA and extensively validated will be used. It solves the FW-H equation (without the volume term) in the time domain according to the formulation developed by Prieur \& Rahier [43], written for a reference frame $\left(\mathbf{e}_{1}, \mathbf{e}_{2}, \mathbf{e}_{3}\right)$ in translation at the velocity $U_{\infty} \mathbf{e}_{1}=M c_{\infty} \mathbf{e}_{1}$ as [39]:

$$
\begin{aligned}
p^{\prime}(\mathbf{x}, t) H(f)=\iint_{\Sigma(f=0)}\left[\left(L_{i} \frac{\partial d}{\partial x_{i}}-Q U_{\infty} \frac{\partial d}{\partial x_{1}}\right)\right. & \left.\frac{1}{4 \pi d^{2}|\partial g / \partial \tau|}\right]_{g=0} \mathrm{~d} S(\mathbf{y}) \\
& +\frac{\partial}{\partial t} \iint_{\Sigma(f=0)}\left[\left(L_{i} \frac{\partial g}{\partial x_{i}}-Q U_{\infty} \frac{\partial g}{\partial x_{1}}+Q\right) \frac{1}{4 \pi d|\partial g / \partial \tau|}\right]_{g=0} \mathrm{~d} S(\mathbf{y})
\end{aligned}
$$

where

$$
L_{i}=p^{\prime} n_{i}+\rho u_{i}\left(u_{n}-v_{n}{ }^{\Sigma}\right), \text { and } Q=\rho_{\infty} v_{n}{ }^{\Sigma}+\rho\left(u_{n}-v_{n}{ }^{\Sigma}\right)
$$

The retarded-time function $g$ is:

$$
g=\tau-t+\frac{d-M\left(x_{1}-y_{1}\right)}{c_{0} \beta^{2}}
$$

And the distance $d$ is evaluated with the Prandtl-Glauert factor $\beta^{2}=1-M^{2}$ according to:

$$
d=\sqrt{C_{i}\left(x_{i}-y_{i}\right)^{2}} \text { where } C_{i}=\left(1-\beta^{2}\right) \delta_{1 i}+\beta^{2}
$$

The expression for the derivatives $\partial d / \partial x_{i}, \partial g / \partial x_{i}, \partial g / \partial \tau$, as well as the corrective terms added to the surface integral in order to mitigate spurious radiation associated to turbulence crossing the surface are detailed in Rahier et al. [39], and are not written here for the sake of conciseness. Please note that Eq. 12 reduces to Eqs 9 and 11 when simplifications are applied for appropriate control surfaces, and that no far-field approximation is made. This means that $O\left(1 / d^{n}\right)$ terms are all taken into account, while far-field approximations (retaining only the $O(1 / d)$ terms) are sometimes used in other studies. This point will be discussed later on.

\section{Description of the test case and flow features}

\subsection{Geometry and computational setup}

\subsubsection{Main dimensions of the LAGOON wheel}

The LAGOON geometry is a 1:2.5 scaled canonical Airbus A320 two-wheel nose landing gear designed in the scope of an Airbus-ONERA collaboration (2006-2010). Three increasingly complex geometries were defined, the first (LAGOON1, see figure 2-left) being the only one fully disclosed in the public domain. The experimental campaign, led by Manoha et al. [44, 45], provided data from ONERA's F2 and C19 wind tunnels that allowed several teams to compare their numerical simulations within the context of the Benchmark problems for Airframe Noise Computations (BANC) [46].

A single wheel from this geometry, without the axle, will be considered in this study. The coordinate system has its origin on the center of the cavity floor. The flow is directed in the $x$-direction, and the $z-$ direction points towards the cavity mouth (see figure 2 -right). According to the classification proposed by Zdravkovich et al. [47], it can be seen as a "coin-like" cylinder of diameter $D_{w}=3 \times 10^{-1} \mathrm{~m}$ and width $H_{w}=9 \times 10^{-2} \mathrm{~m}$, inside of which is installed a shallow round cavity of internal radius $r=81 \times 10^{-3} \mathrm{~m}$ and depth $h=37 \times 10^{-3} \mathrm{~m}$. Circular cavities can be classified according to their depth-to-diameter ratio 

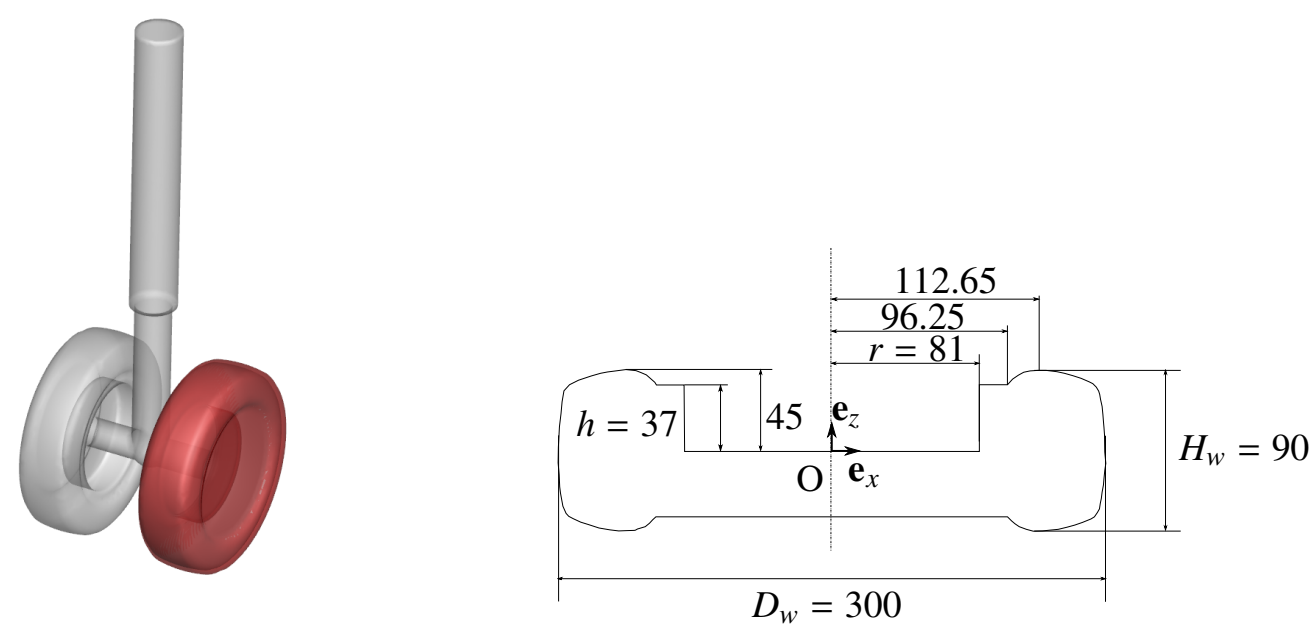

Figure 2: LAGOON geometry (left) and cut view of the isolated wheel in a median plane with the main dimensions in mm (right)

$\kappa$. In the case of the LAGOON cavity, this parameter has a value $\kappa=0.23$, which ranks it in the shallow cavities category. The cavity being different from an academic configuration on an infinite plane, $\kappa$ may vary between 0.17 and 0.28 depending on the values chosen for $h$ and $r$, but still remains inferior to unity.

A common feature of cavity flows is that they often induce a strong tonal response in the far-field. This tonal response can originate either from a feedback phenomenon where sound waves are generated at the downstream edge of the cavity and interact with the shear layer at its detachment point, or by an interaction between the shear layer instability modes and the depth modes of the cavity. The first feedback model has been described by Rossiter [48] for rectangular cavities, but its relevance for cylindrical cavities is less evident due to the varying length of the cavity in the crossflow direction. Marsden et al. [49] have performed experiments on several cavities with depth to diameter ratio between 1 and 1.5 and found that tonal emission resulted from an interaction between the shear layer and the first depth mode of the cavity. The same authors then performed Large Eddy Simulations of circular cavities with $\kappa \leq 1$ [50] and stated that no strong tone could be expected in the far-field for such $\kappa$ values, which is in accordance with a qualitative reasoning on the quality factor of shallow rectangular cavities proposed by Heller et al. [51]. They also provided a detailed analysis of the mean flow regimes that can occur in such cavities. A notable difference in the case of the LAGOON wheel is that the shear layer detaches on the rounded tire, as opposed to grazed academic cavities [34].

The upstream conditions are that of the LAGOON experiment in the CEPRA19 (ONERA's anechoic wind tunnel) configuration, namely $P_{\infty}=96772.3 \mathrm{~Pa}, T_{\infty}=288.39 \mathrm{~K}, \rho_{\infty}=1.18 \mathrm{~kg} / \mathrm{m}^{3}$ and $M_{\infty}=0.23$. In these conditions, the Reynolds number based on the wheel diameter is about $R e_{D_{w}}=1.56 \times 10^{6}$.

\subsubsection{Navier-Stokes setup}

Computational domain and numerics.

For the present computations, ONERA's in-house code CEDRE [52] is used to numerically solve the full compressible Navier-Stokes equations around the wheel. CEDRE is a cell centered, unstructured, finite volume solver originally developped for multi-physics applications. Its use for compressible aeroacoustic applications is more recent but has already given very satisfying results on the LAGOON test case [7]. The computational domain is a rectangular box of dimensions $\left(L_{x} / D_{w}, L_{y} / D_{w}, L_{z} / D_{w}\right)=(40,24,18)$. Turbu- 
lence is handled by performing a Zonal Detached Eddy Simulation (ZDES) mode II based on the subgrid length scale $\Delta_{\omega}$ and the Spalart-Allmaras one-equation turbulence model in its fully turbulent ("No- $f_{t 2}$ ") version. For further information about the definition of the RANS and DES zones within the ZDES method, the reader can refer to [53]. A 2nd order implicit Runge-Kutta scheme is used for temporal integration, associated with a GMRES algorithm. The flux calculations are performed with a 2nd order MUSCL method and an HLLC scheme. Spurious reflexions at the boundaries of the computational domain are avoided by stretching the CFD mesh in all directions and with the use of a characteristic-based non-reflective boundary conditions.

The calculations have been initialized in URANS mode during 55.78 convective times based on the wheel diameter $T_{D_{w}}=D_{w} / U_{\infty}$ before the ZDES was started. Then, after a ZDES transient period, the flow has been solved for a total timelength of $328 \mathrm{~ms}\left(85.5 T_{D_{w}}\right)$. As for the computation of the power spectral densities, the Welch estimator is employed by windowing and averaging overlapping blocks with a Hann function (66\% of overlap), to obtain a target Strouhal number resolution $\Delta S t_{D_{w}}=0.115$.

\section{Computational grids description.}

A view of the mesh used in this work is presented in figure 3 in the $y=0$ plane. It consists in a hybrid

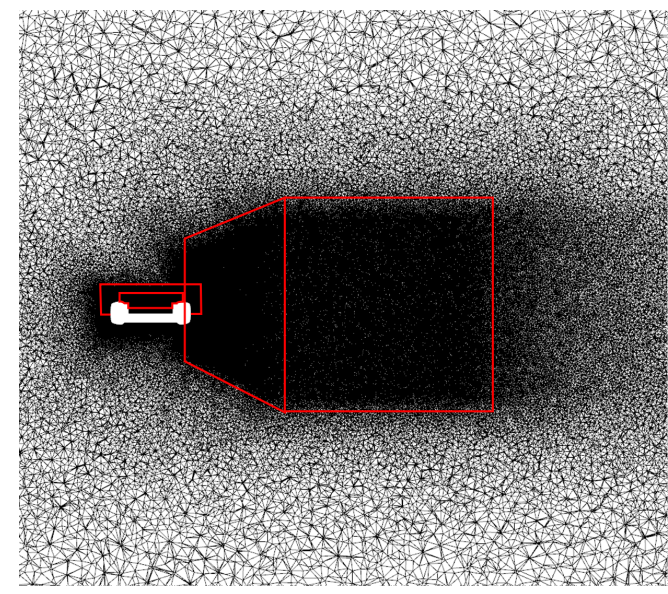

Figure 3: Cut of the baseline grid in a median plane of the wheel. The red lines delineate the different refinements.

unstructured grid that includes 3.7 million prisms (21 layers, with a first cell height of $3.33 \times 10^{-5} D_{w}$, leading to $y^{+}$values of no more than 2) devoted to solving the attached boundary layers at the wheel surface, except on the cavity floor $\left(y^{+} \simeq 35\right)$, where a wall law is applied. The rest of the domain is discretized with tetrahedra exclusively, of which characteristic size $\Delta$ is defined as the diameter of the tetrahedron inscribed sphere. This characteristic size conditions the mesh acoustic cutoff according to the following relationship:

$$
S t_{D_{w}}{ }^{\max }=\frac{\left(c_{0} \pm U_{\infty}\right) D_{w}}{\text { PPW } \Delta U_{\infty}}
$$

Pure acoustic propagation tests carried out at ONERA with the same numerical schemes as used in this work have shown that a number of points per wavelength PPW $=20$ was required to propagate acoustic waves with an acceptable dissipation error [24].

Two concentric volume refinements, depicted in fig. 3 with red rectangles over the cavity, are present in order to accurately resolve the shear layer that detaches on the rounded part of the upstream tire. The smallest one is defined by $0.54 \leq z / h \leq 1.76$ and $\sqrt{x^{2}+y^{2}} / r \leq 1.42$, with an imposed cell size $\Delta / D_{w}=$ 
$2 \times 10^{-3}$. The larger one is defined by $0 \leq z / h \leq 2.16$ and $\sqrt{x^{2}+y^{2}} / r \leq 2.22$, with $\Delta / D_{w}=3.4 \times 10^{-3}$. Two more refinements have been added downstream of the wheel to obtain an accurate resolution of the wake, as well as a good propagation of the acoustic waves in anticipation of the permeable surface computations. The first one is of trapezoidal shape, the smaller base being in the $x / D_{w}=0.4$ plane and covering the intervals $\left|y / D_{w}\right| \leq 0.8$ and $-0.3 \leq z / D_{w} \leq 0.6$. The larger base is located about one wheel diameter away from the smaller one in the plane $x / D_{w}=1.6$ and covers the intervals $\left|y / D_{w}\right| \leq 0.8$ and $-1 \leq z / D_{w} \leq 1.2$. The tetrahedra composing this refinement are slowly (with a constant linear rate $\mathrm{d} \Delta / \mathrm{d} x$ ) stretched from the smaller base, where their size is $\Delta / D_{w}=3.4 \times 10^{-3}$, towards the larger base where the cell size reaches a constant value $\Delta_{0} / D_{w}$. Downstream of this refinement is located a last box-shaped zone, which consists in the extrusion in the $\mathbf{e}_{x}$ direction of the larger base from $x / D_{w}=1.6$ to $x / D_{w}=3.8$. The cell size imposed is kept equal to $\Delta_{0} / D_{w}$. Then, the grid is stretched in the $\mathbf{e}_{x}$ direction with a rate $\mathrm{d} \Delta / \mathrm{d} x=5 \times 10^{-3}$ up to the end of the domain.

In order to assess the grid convergence in the wake region, three grids have been generated by varying the stretching rate in the trapezoidal refinement as well as the cell diameter in the box-shaped refinement. The numerical values corresponding to the three grids are presented in Tab. 1, and the corresponding evolution of the cell diameter in the streamwise direction is depicted in figure 4. The cell size evolution in the wake of the full LAGOON computation [7] has also been added for comparison. Please note that in the latter case, off-body RMS velocity fields were already pretty well resolved with respect to the experimental data. The grid was also quickly stretched after $x / D_{w}=2.5$ as only solid-surface results were targeted. In the present case, the very fine resolution of the wake sources is responsible for a large proportion of the total cell count. The time step of the simulation has been adapted in the three simulations in order to keep an acoustic CFL number below 0.8 in the wake region.

\begin{tabular}{cccccccc}
\hline & $\begin{array}{c}\mathrm{d} \Delta / \mathrm{d} x\left(\times 10^{-3}\right) \\
0.4<x / D_{w}<1.6\end{array}$ & $\begin{array}{c}\Delta_{0} / D_{w}\left(\times 10^{-3}\right) \\
1.6<x / D_{w}<3.8\end{array}$ & Cell count $\left(\times 10^{6}\right)$ & $\Delta t(\mu \mathrm{s})$ & Time steps & $\mathrm{CFL}$ & $\begin{array}{c}S t_{D_{w}}{ }^{\max } \\
\text { Upst. - Downst. }\end{array}$ \\
\hline coarse & 5 & 9.4 & 48 & 6.45 & 50803 & 0.78 & $18-29$ \\
baseline & 3.25 & 7.3 & 60 & 5 & 65536 & 0.78 & $23-37$ \\
fine & 1.6 & 5.2 & 90 & 3.5 & 93714 & 0.76 & $32-52$ \\
\hline
\end{tabular}

Table 1: Grids used to assess the convergence of the CFD solution

According to equation 16, the acoustic cutoff Strouhal number can be estimated for upstream and downstream traveling waves. On the baseline grid, the maximum resolved Helmholtz number $M S t^{\mathrm{max}}$ lies then between 5.4 and 8.5 which means that, based on its diameter, the wheel considered in this study will be non compact for the highest frequencies supported by the mesh. Please note that, as the width-to-diameter ratio of the LAGOON wheel is about 0.3 , the previously cited $M S t^{\max }$ values also remain superior to unity if the ${ }_{255}$ wheel width is considered as the reference length.

\subsection{Aerodynamic analysis}

A thorough analysis of the mean and unsteady flow results inside the cavity has already been carried out in [34] and will not be recalled here for the sake of brevity. This analysis allowed to conclude that the LAGOON shallow circular cavity behavior compares well with the literature of grazing cavities as for the development of the shear layer, as well as the wall pressure inside the cavity. Some additional aerodynamic features are presented in the following. 


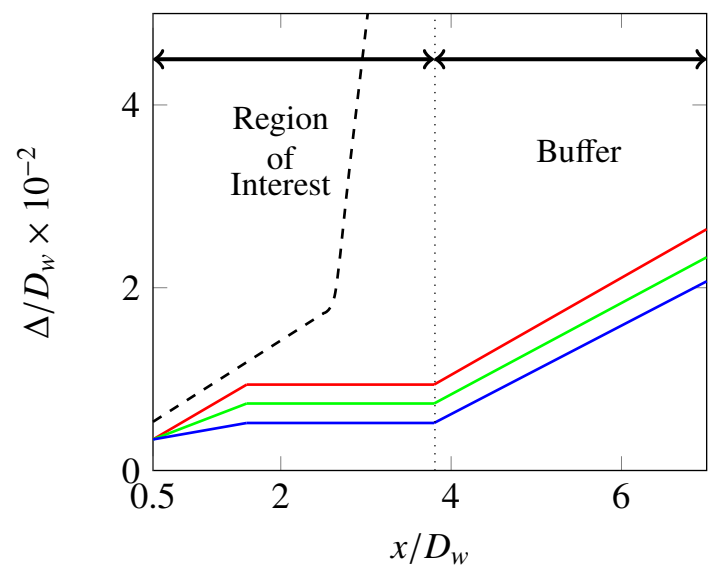

Figure 4: Cell size $\Delta / D_{w}$ evolution downstream of the wheel centreline $(y=z=0)$. ( - - ): LAGOON G2 [7], (一): coarse, ( -): baseline, (-): fine.

\subsubsection{Mean azimuthal $C_{p}$ variation}

Experimental works from Lazos [54, 55, 56] have highlighted the complexity of the surface flow on the wheels of the Simplified Landing Gear thanks to oil-flow visualizations. In the case of an isolated wheel, the flow topology is simplified due to the absence of interaction with another downstream wheel, but still presents some interesting features that can be related to experimental measurements of Zdravkovich et al. [47] on small aspect ratio ("coin-like") cylinders, or previous experiments performed at the University of Southampton on the CADWIE prototype by Zhang et al. [57]. For instance, the mean and RMS pressure coefficients, defined as:

$$
\overline{C_{p}}=\frac{\bar{p}-P_{\infty}}{0.5 \rho U_{\infty}^{2}}, \quad C_{p_{\mathrm{rms}}}=\frac{p_{\mathrm{rms}}}{0.5 \rho U_{\infty}^{2}}
$$

are presented in figure 5 for the present computation, along with experimental measurements of Zhang et al. and Zdravkovich et al.

First, we can notice that, despite the presence of a hub cavity in the CADWIE case, the measured $\overline{C_{p}}$ distribution follows that of Zdravkovich et al. on an academic cylinder with the same aspect ratio. The LAGOON wheel has a width-to-diameter ratio of 0.3 , for which no experimental measurements are available. However, the trend highlighted by Zdravkovich et al. seems to indicate that the minimum $\overline{C_{p}}$ increases with decreasing aspect ratio, in coherence with the present computation. After this minimum is reached, our results lie between the measurements of Zdravkovich et al. for aspect ratios of 0.23 and 0.39 , which is the expected behavior. Contrarily to the typical plateau observed on a nominally $2 \mathrm{D}$ cylinder, an oscillation is observed in the rear part of the wheel, around $\theta=145^{\circ}$, as already reported by Lazos [54].

A second common observation with those made by Lazos is the existence of several possible mean flow states downstream of the wheel. Indeed, while the $\overline{C_{p}}$ distributions on the coarse and fine grids are stricly identical (see figure 5-top), the baseline computation presents a symmetrical $\overline{C_{p}}$ evolution with respect to the coarse and fine simulations. To illustrate this phenomenon, the same figure has been replicated in figure 5-bottom with the $\theta$ angle being counted clockwise only for the baseline grid. The three mean distributions then collapse perfectly. As for the RMS distributions, the agreement is satisfying between the baseline and the fine grids, showing that grid-independence is achieved as far as the surface pressure on the wheel is 280 concerned. 

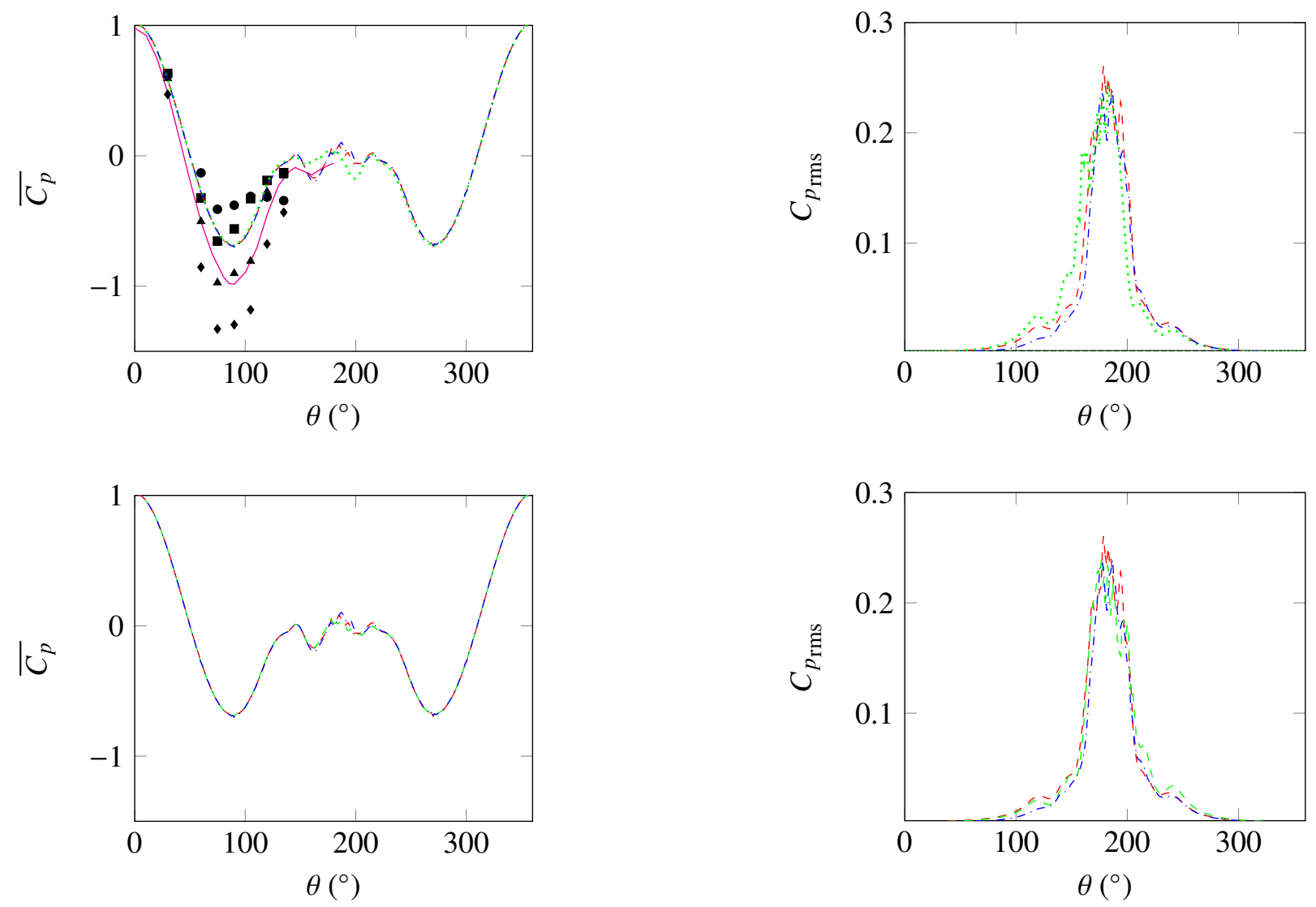

Figure 5: Mean and RMS pressure coefficient azimuthal evolution in the $z=0$ plane. (- - ): coarse, ( $\cdots)$ : baseline, (- - -): fine, $L / D=0.3$ (Num.), (一): Zhang et al. [57] $L / D=0.39$ (Exp.), $\bullet / \mathbf{\square} / \mathbf{\Delta} / \bullet:$ Zdravkovich et al. [47] $L / D=0.11 / 0.23 / 0.39 / 0.63$ (Exp.). Top: $\theta$ orientation identical for the three simulations. Bottom: $\theta$ orientation reversed for the baseline grid only.

\subsubsection{Surface streamlines}

Surface streamlines on the wheel surface, computed from the baseline grid, are presented in figure 6 from several perspectives, showing a very similar topology as that proposed in Zdravkovich et al. [47]. Detachments due to adverse pressure gradient take place on both sides of the wheel due to the tire curvature. A small recirculation bubble is readily noticeable on the external side of the cavity, while the streamlines orientation on the other side highlights the recirculation that occurs inside the circular cavity. Tridimensional effects appear on the side views as a consequence of the finite width of the wheel, showing how a portion of the mean flow is pushed on the external faces, while another part meets in the rear, leading to the formation of two counter-rotating vortices with axis aligned with the flow direction. The spacing between these vortices pairs is different, depending on which side of the wheel is considered. On the cavity side, the distance is greater than on the external side. The vortex core positions on the rear part of the wheel are very similar in the three computations, and are summarized in Table 2

\subsubsection{Instantaneous and mean flow}

Having examined the mean flow topology on the wheel surface, we now turn to the investigation of the turbulent flow around and downstream of the wheel. Figure 7 shows a superposition of the instantaneous norm of the vorticity vector $\Omega$, with a color scale such that $19500 \leq \Omega U_{\infty} / D_{w} \leq 520500$, and the pres- 


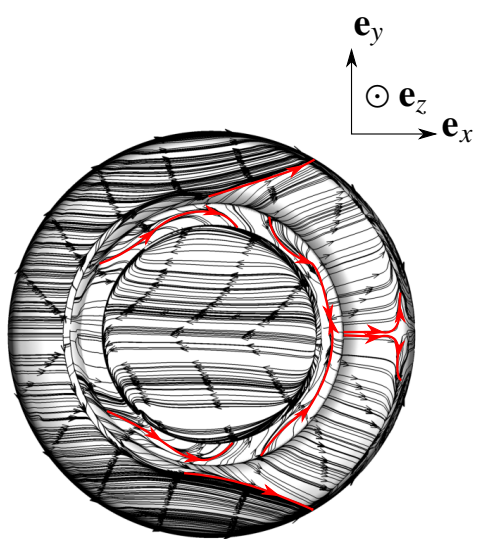

Top

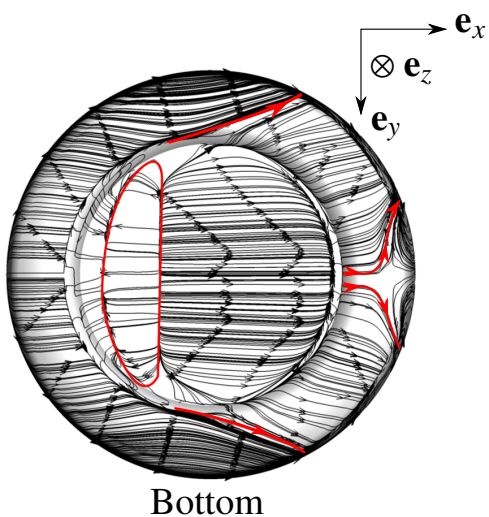

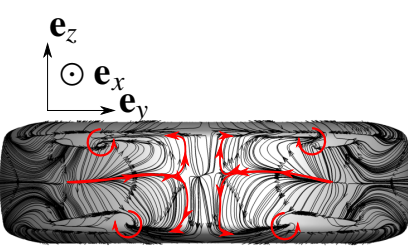

Rear

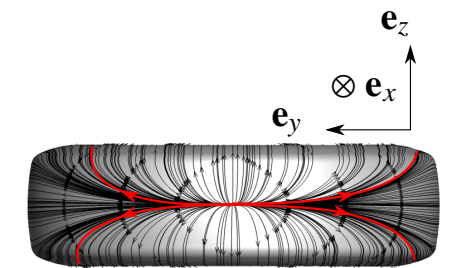

Front

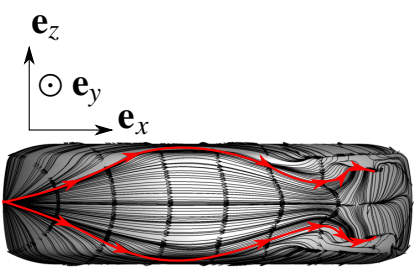

Side

Figure 6: Surface streamlines on the LAGOON wheel. Baseline grid resuls. Thick red lines delineate the mean flow organization.

\begin{tabular}{cccc}
\hline & $\begin{array}{c}x / D_{w} \\
\text { Cav. } / \text { Ext. }\end{array}$ & $\begin{array}{c}y / D_{w} \\
\text { Cav. / Ext. }\end{array}$ & $\begin{array}{c}z / D_{w} \\
\text { Cav. / Ext. }\end{array}$ \\
\hline coarse & $0.41 / 0.44$ & $\pm 0.28 / \pm 0.20$ & $0.10 /-0.11$ \\
baseline & $0.42 / 0.45$ & $\pm 0.25 / \pm 0.19$ & $0.10 /-0.10$ \\
fine & $0.42 / 0.45$ & $\pm 0.25 / \pm 0.20$ & $0.10 /-0.10$ \\
\hline
\end{tabular}

Table 2: Vortex core positions on the rear part of the wheel.

sure gradient magnitude $|\nabla p|$ on an arbitrary gray scale, thus reproducing a numerical Schlieren method. The examination of the vorticity field provides a qualitative feeling for the eddy size and highlights how fine-scale turbulence seems to be well resolved in the wheel wake. Wavefronts are clearly discernable from the pressure gradient magnitude visualization, to the extent permitted by the mesh local size. Large wavelengths originating from the wheel do not seem to be reflected by the computational boundaries, while small wavelengths associated with the turbulent motion in the wheel wake are clearly visible in the resolved area, giving confidence in the adequate design of the grid in the wake.

Iso-surfaces of the $Q$ criterion, defined as $Q=\frac{1}{2}\left[\left(\frac{\partial v_{i}}{\partial x_{i}}\right)^{2}-\frac{\partial v_{i}}{\partial x_{j}} \frac{\partial v_{j}}{\partial x_{i}}\right]$ and colored by the local velocity 305 magnitude, are given in figure 8 for a value $Q D_{w}{ }^{2} / U_{\infty}{ }^{2}=1.5 \times 10^{-3}$. Comparing the two faces of the wheel, we can observe the presence of hairpin vortices forming on the external face (figure 8-left), while 

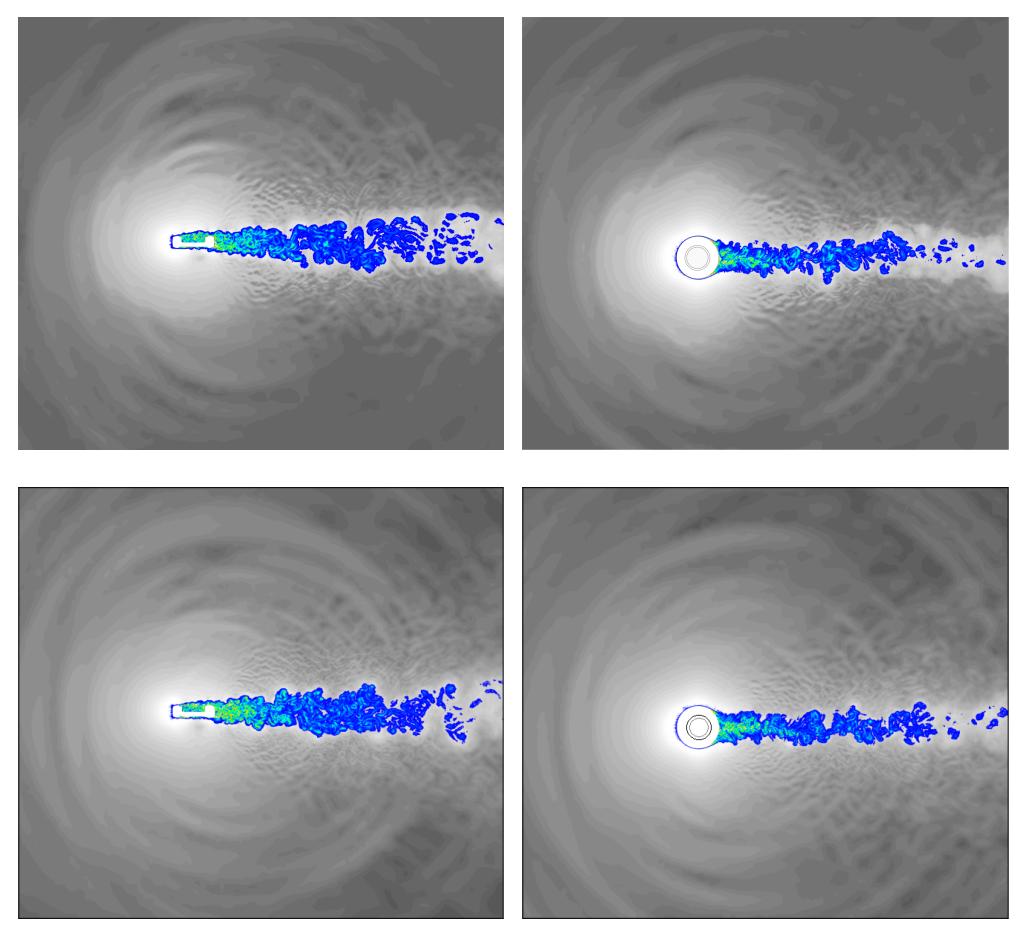

Figure 7: Instantaneous contours of the vorticity magnitude (colorscale: $19500 \leq \Omega U_{\infty} / D_{w} \leq 520500$ ) and pressure gradient magnitude $|\nabla p|$ (gray arbitrary scale). Top: baseline grid, bottom: fine grid. Left: $x O z$ plane, right: $x O y$ plane.

on the cavity side (figure 8 -right), the formation of rollers stretched in the $\mathbf{e}_{y}$ direction reflect the KelvinHelmholtz instabilities associated with the flow separation on the upstream part of the tire. Again, fine-scale turbulence seems well resolved around the wheel, as well as in the wake forming just downstream.

The mean flow in the wake region is presented in a more quantitative way by looking at the velocity profiles $\bar{U} / U_{\infty}$ inside the refined region at six equally spaced planes downstream of the wheel, from $x=D_{w}$ to $x=3.5 D_{w}$, as presented in figure 9. The typical Gaussian-shaped velocity profile is observed in the $\mathbf{e}_{y}$ direction. The mean velocity deficit rapidly declines as the wake tends to contract in that direction. On the other hand, in the $\mathbf{e}_{z}$ direction, the wake expands for increasing $x$ while becoming more axisymmetric. A 315 notable point is that the first velocity profiles exhibit a higher flow velocity (smaller deficit) on the $z \geq 0$ (cavity) side. Zdravkovich et al. [47] reported that the free ends of coin-like cylinders considerably increase the total drag, which could explain the profiles shape. The cavity is also expected to contribute to the total drag, but the presence of the recirculating flow, as well as the early detachment of the shear layer might somehow alleviate this drag increase, compared to the external side. Once again, grid convergence seems reached on the baseline grid, although some small discrepancies remain in the velocity profiles for this mesh resolution. These discrepancies are most likely due to the different mean flow state already observed through the examination of the pressure coefficient distribution.

The wake displacement and momentum thicknesses have been computed at the six same locations according to the following formulas:

$$
\delta^{*}=\int_{-\infty}^{+\infty}\left(1-\frac{U(\alpha)}{U_{0}}\right) \mathrm{d} \alpha, \quad \theta=\int_{-\infty}^{+\infty} \frac{U(\alpha)}{U_{0}}\left(1-\frac{U(\alpha)}{U_{0}}\right) \mathrm{d} \alpha
$$

The results are presented in figure 10 where the integration of the velocity profiles have been performed 

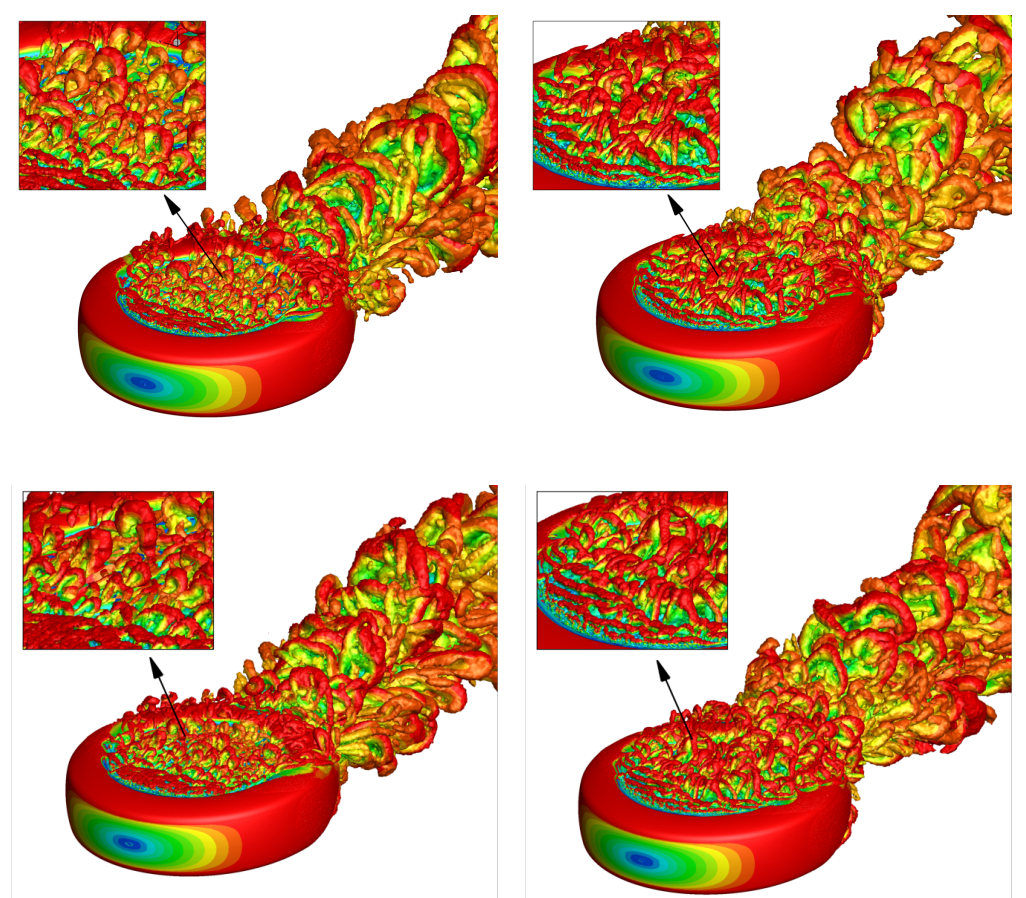

Figure 8: Iso-surfaces of the Q-criterion for a value $Q D_{w}{ }^{2} / U_{\infty}{ }^{2}=1.5 \times 10^{-3}$ colored by the local flow velocity. Top: baseline grid, Bottom: fine grid. Left: external side of the wheel $(z \leq 0)$, right: cavity side $(z>0)$.
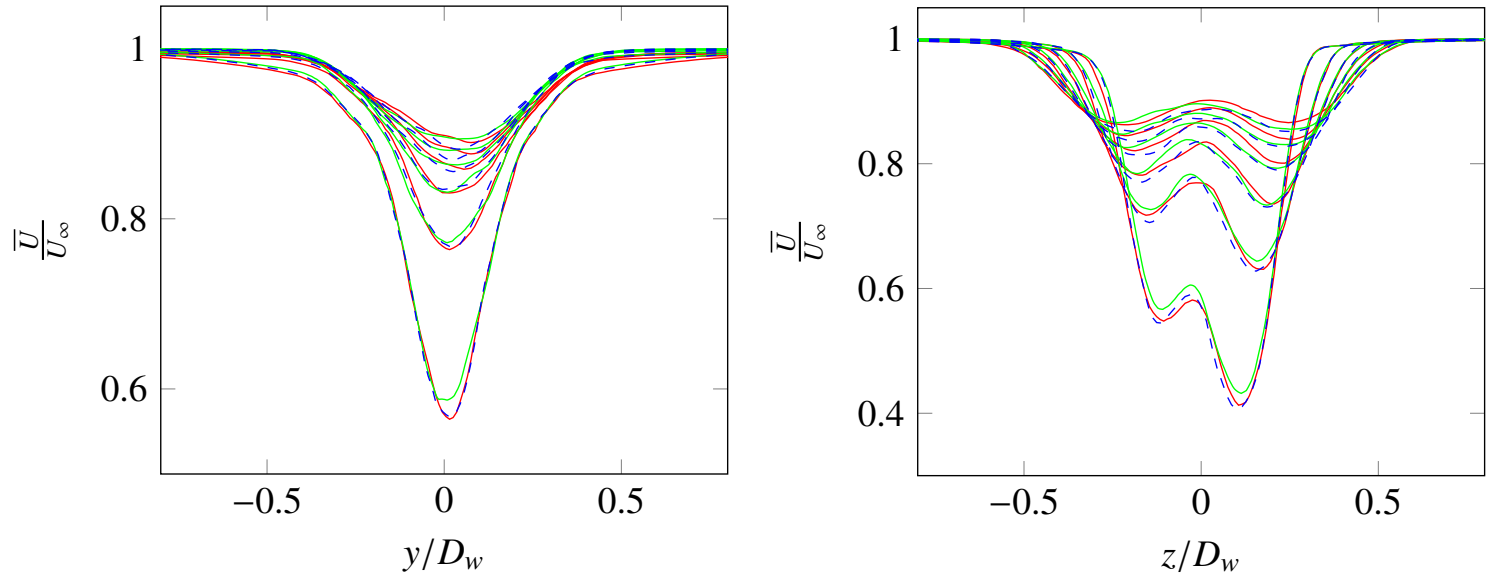

Figure 9: Mean streamwise velocity profiles in the wheel wake. Left: in the crosswise direction, right: in the transverse direction. (一): coarse, (-): baseline, (- - ): fine. Profiles from $x / D_{w}=1$ to 3.5 by step of 0.5 .

along the $y$ and $z$ directions separately. In both directions, despite the small differences that appeared locally 325 in the velocity profiles, we find that when integrated on the entire wake transverse directions, the mean axial velocity is perfectly converged between the baseline and the fine grids.

As for second-order statistical moments, the resolved turbulent kinetic energy contours are presented in figure 11 for the three grids, showing very similar distributions with a very clear TKE maximum just downstream of the wheel, closer to the external side than to the cavity side. 

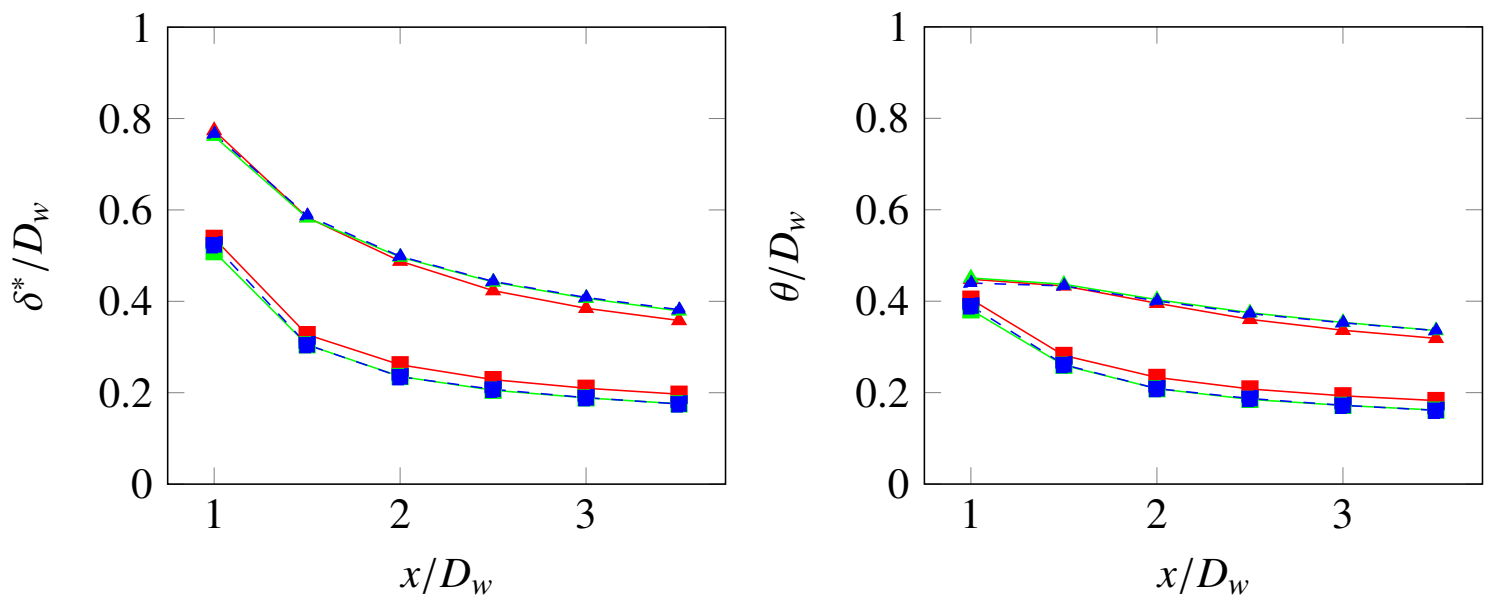

Figure 10: Wake displacement (left) and momentum (right) thicknesses corresponding to the mean velocity profiles presented in figure 9 (一): coarse, (-): baseline, (- - ): fine. $\mathbf{\square}$ : integration along the $y$-direction, $\mathbf{\Delta}$ : integration along the $z$-direction.

Power spectral densities of the crosswise and transverse velocity components are depicted in figure 12 for the same six $x$ positions in the wheel wake, on the $y=z=0$ centreline. The spectra have been plotted every $20 \mathrm{~dB}$ to improve readability. The $S t^{-5 / 3}$ slope is also represented by a dashed line for indicative purpose. Globally, as one would expect, the turbulent energy carried by the flow decreases significantly with the distance, and faster with the coarse grid than with the fine one. The inertial subrange is also logically increased with the grid resolution in proportions that roughly correspond to the cell diameter $\Delta_{0} / D_{w}$ in the wake region. No particular Strouhal number signifying a regular shedding is observed due to the highly three-dimensional character of the flow.
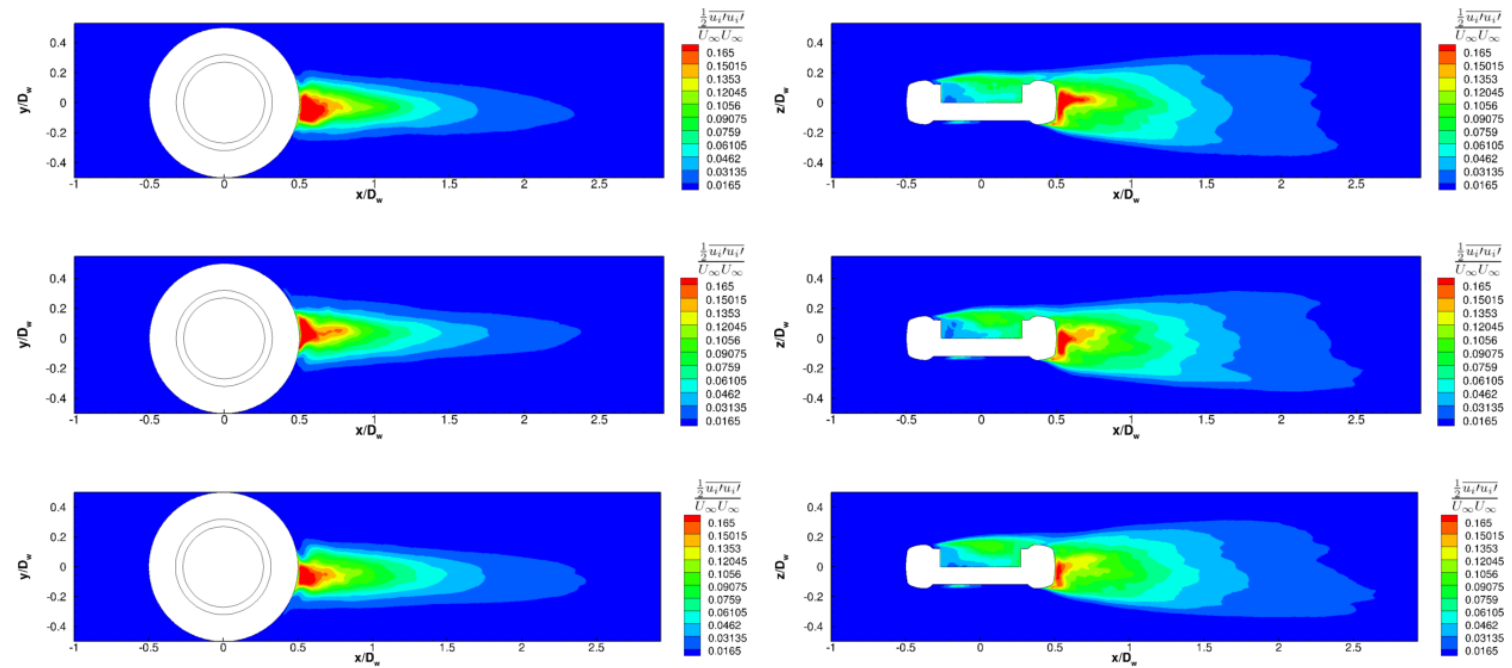

Figure 11: Contours of the resolved turbulent kinetic energy. Left: $x O y$ plane, right: $x O z$ plane. Top-to-bottom: coarse, baseline, fine grid.

In addition to the aerodynamic quantities, the near-field noise computed directly by the compressible CFD solver has been examined in the vicinity of the wake, at the frontier of the box-shaped refinement 

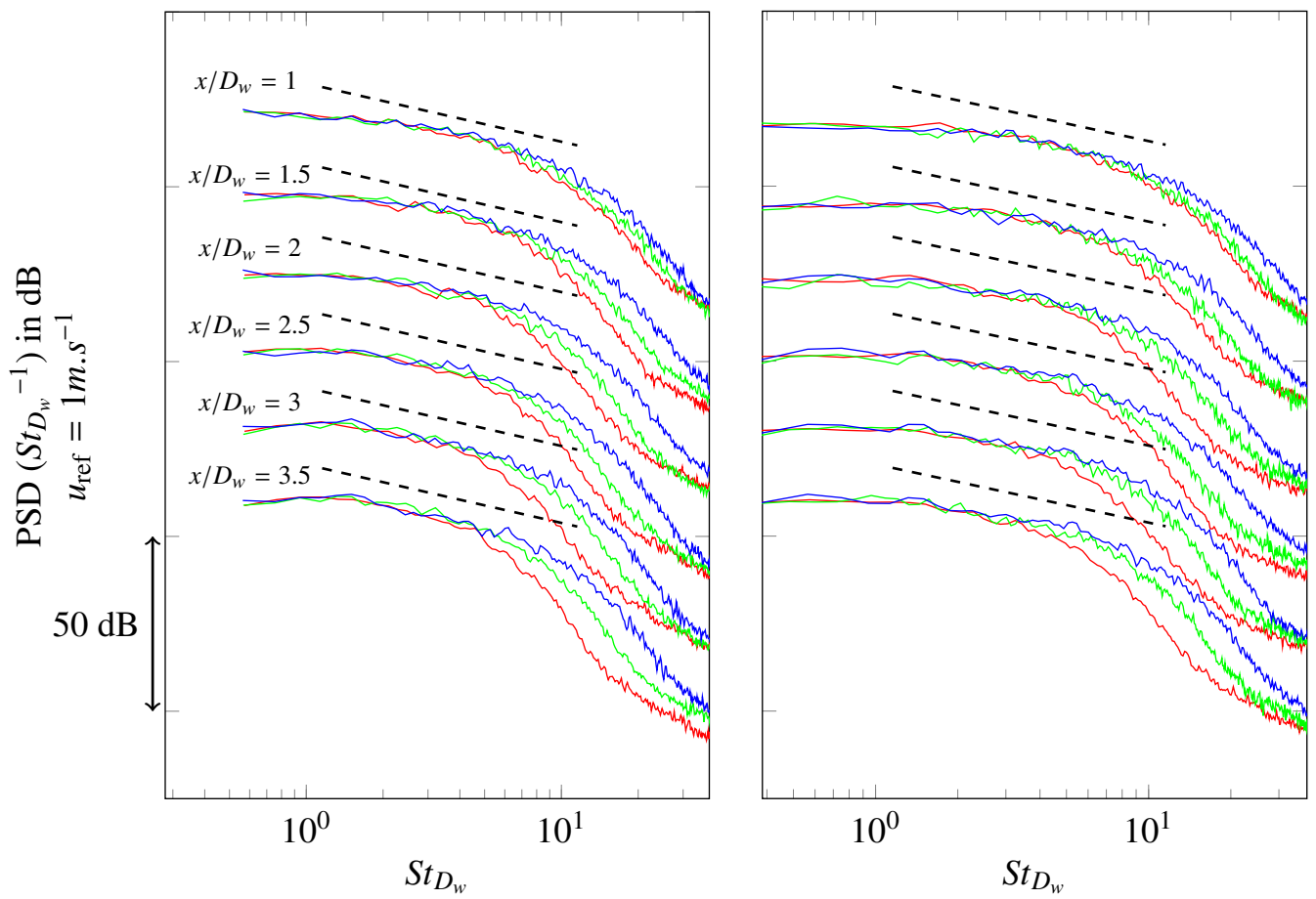

Figure 12: Power spectral density of $u_{2}$ (left) and $u_{3}$ (right) at six positions $x / D_{w}$ in the wheel wake. (一): coarse, (一): baseline, (一): fine. (- - -): Kolmogorov's $S t^{-5 / 3}$ power law.

located downstream of the wheel. The pressure spectrum for an observer located at $\left(x / D_{w}, y / D_{w}, z / D_{w}\right)=$ $(2.7,0,1.2)$, obtained with the direct compressible Navier Stokes solver is thus plotted in figure 13 for the three grid resolutions. First, the extension of the mesh acoustic cut-off appears evident between the three

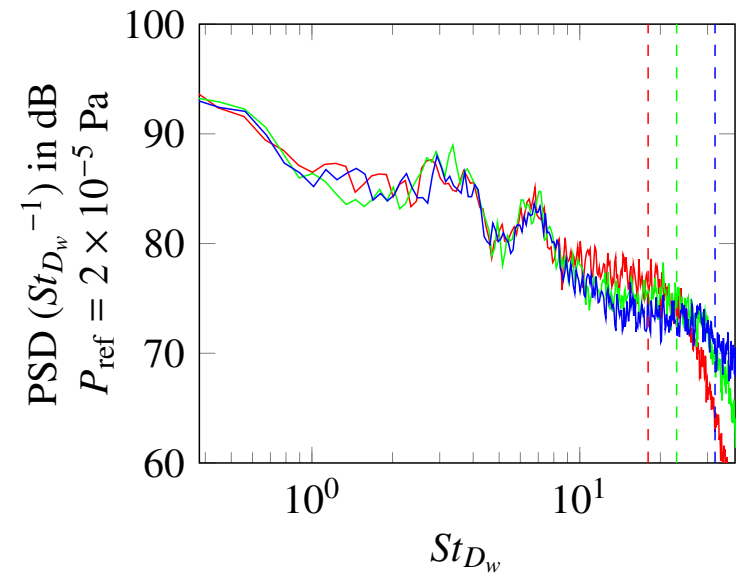

Figure 13: Power spectral density of the pressure fluctuations computed by the Navier-Stokes compressible solver at $\left(x / D_{w}, y / D_{w}, z / D_{w}\right)=(2.7,0,1.2)$. (一): coarse, (一): baseline, (一): fine. Dashed vetical lines: theoretical 20PPW cut-off frequencies according to eq. 16

grids: the levels are seen to rapidly fall off on the coarse grid around $S t_{D_{w}}=18$, while the fall off observed 
on the baseline mesh (with, seemingly, the same $S t$-decay), is seen to occur around $S t_{D_{w}} \simeq 23$, in coherence with the cut-off estimated with Eq. 16. One can clearly discern a frequency hump around $S t_{D_{w}}=7.1$, which does not correspond to any of the cavity natural resonant modes. The origin of this pseudo-tonal component is still not well understood, and a second run at another Mach number could help to identify the underlying mechanism. On the other hand, despite small differences (about 1-2 dB at most) at the highest resolved Strouhal numbers, the differences in noise levels computed on the baseline and fine grid indicate that the quadrupole sources within the wake, as well as their propagation, should be adequately resolved on the baseline grid. Therefore, in the rest of the paper, only data extracted from the baseline simulation will be used for the acoustic computations.

\section{Computation of the far-field}

\subsection{Ffowcs-Williams $\mathcal{E}$ Hawkings setup}

For all our calculations of narrow-band spectra, the observers are located in the wheel median plane $x O z$, which would correspond to a pure sideline plane on a real landing gear configuration. These points describe a circle around the wheel center, with an angular resolution $\Delta \varphi=2^{\circ}$. OASPL polar plots will also be displayed in the $y O z$ plane. The observer distance $R_{\text {obs }}$ influence, will be addressed by considering four different radii $R_{\mathrm{obs}}=5,10,50,100 D_{w}$. For the sake of brevity, four particular angular positions have been selected, noted hereinafter P1, P2, P3 and P4. P2 $\left(\varphi=+30^{\circ}\right)$ and P3 $\left(\varphi=-30^{\circ}\right)$, mutually symmetrical with respect to the cavity normal direction $\left(\varphi=0^{\circ}\right)$, are expected to give information about the potential tonal response of the cavity. In particular, P2 was already identified as the cavity tonal dominant direction in the solid computation of De la Puente et al. [34]. P1 $\left(\varphi=+80^{\circ}\right)$ and P4 $\left(\varphi=-80^{\circ}\right)$, have been selected as grazing angles are preferentially influenced by spurious noise, or effects of permeable surface opening. As such, they should provide a good estimation of the corrective terms and surface opening effects, respectively. A sketch of the observer positions is proposed in figure 14-top left.

A closed permeable FW-H data surface $\Sigma$ containing the wheel and a part of its wake has been defined in line with the mesh definition. Its shape approximately follows the expansion of the wake and extends from $x=-0.8 D_{w}$ to $x=3.8 D_{w}$. In the crossflow direction, the dimension of the surface is $1.6 D_{w}$. A sketch of the permeable FW-H surface is presented in figure 14-top right, along with its dimensions. In order to investigate the influence of the surface discretisation, three cases are defined: a "fine" surface, with quadrilateral surface elements of size $\Delta_{\Sigma}$ such that $D_{w} / \Delta_{\Sigma}=60$, a medium and a coarse one, obtained by successively coarsening the surface elements by a factor 2 , such that:

$$
\frac{D_{w}}{\Delta_{\Sigma}}= \begin{cases}60 & (\text { fine }) \\ 30 & \text { (medium) } \\ 15 & \text { (coarse) }\end{cases}
$$

The solid integration surface corresponds exactly to the CFD surface mesh, composed of triangles only. Both solid and permeable surfaces are shown in figure 14-bottom. In both cases, for the storage of the data on the integration surface, linear interpolation is performed as each CFD iteration from the CFD mesh to 370 the FW-H mesh.

\subsection{Temporal convergence of the spectra}

An improvement of the present computations with respect to the one performed by De la Puente [34] is the extension of the simulation duration. The statistical convergence of far-field noise spectra is assessed 

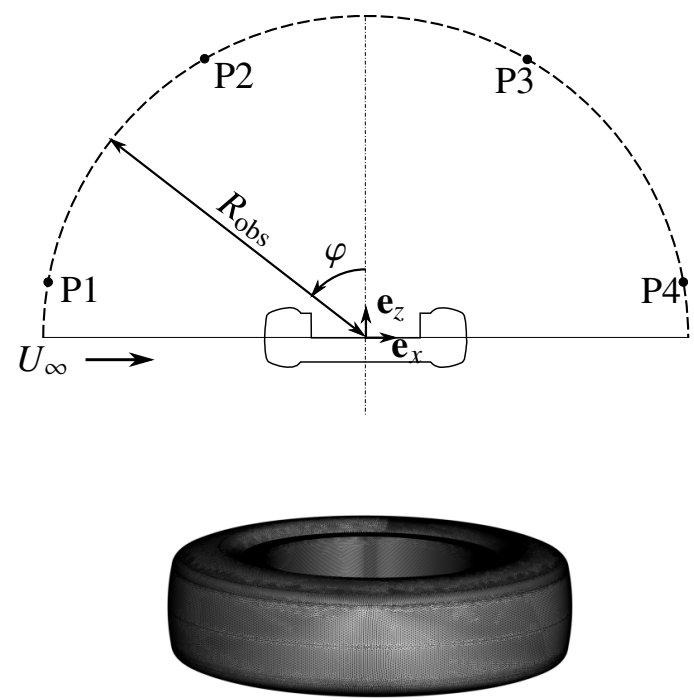
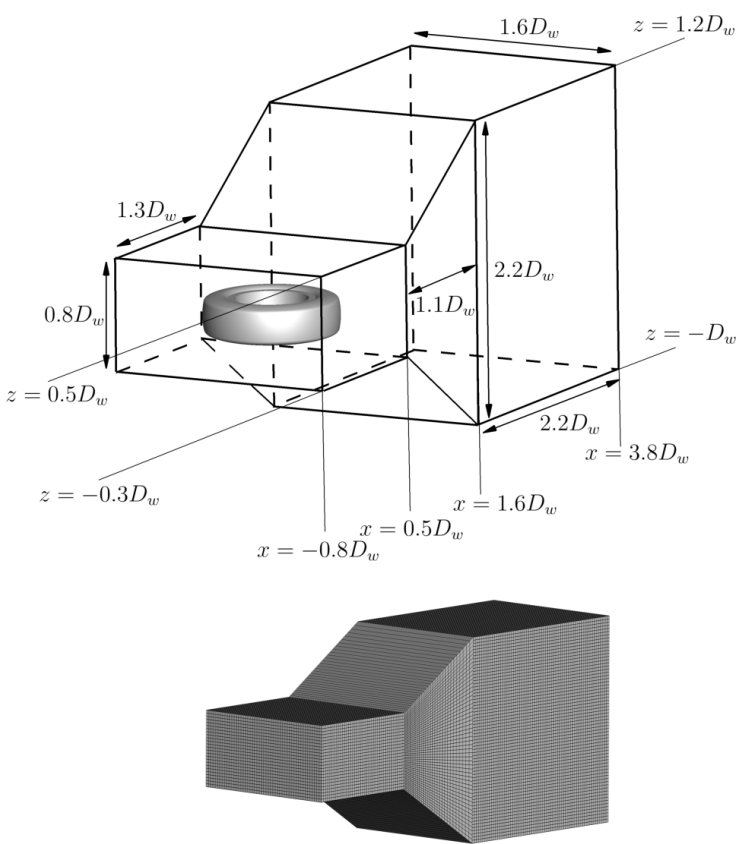

Figure 14: Description of the FW-H setup: observer locations (top-left) and permeable surface (top-right) used in the computations. Bottom: FW-H surface grids.

by comparing the pressure power spectral density (PSD), computed with the solid formulation at the P2 ob- 375 server, using three timelengths referred to as $T_{1}$ (used in De la Puente et al. [34]), $T_{2}$ (present computation), and $T_{3}\left(T_{1}+T_{2}\right)$, equal to $33.25,85.20$ and $118.45 T_{D_{w}}$, respectively. The results are displayed in figure 15 where the dashed vertical line corresponds to a frequency $f=1571 \mathrm{~Hz}$ (resonant mode of the cavity of order (011)). The increase in time duration from $T_{1}$ to $T_{2}$ has intensified the tone at $1.5 \mathrm{kHz}$, which still remains far from the classical tones usually observed in academic cavity studies, as the peak is about 400 $\mathrm{Hz}$ wide.

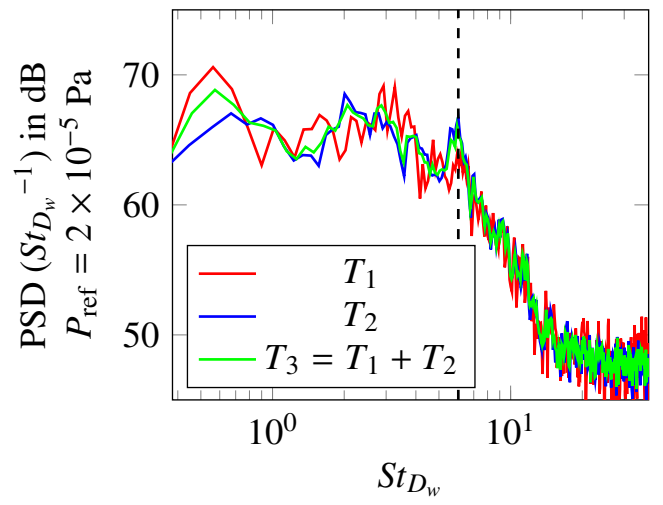

Figure 15: Assessment of the pressure PSD time duration sensitivity at P2 observer with the solid formulation. Dashed line: cavity mode of order (011).

The $T_{3}$ spectrum is almost identical to the $T_{2}$ one for $S t_{D_{w}} \geq 0.8$, showing that a $T_{2}=328 \mathrm{~ms}$ integration time is enough in the present case to obtain converged spectra. We suppose that this conclusion holds 
for the permeable formulation and other angles, and retain this time duration for all FW-H calculations. Additionally, in what follows and unless specified, integrated levels will be calculated for $0.8 \leq S t_{D_{w}} \leq 23$, the lower bound being limited to 0.8 by the available $328 \mathrm{~ms}$ of time signals, and the upper bound by the CFD mesh cut-off frequency previously indicated. Narrowband pressure spectra will be presented for $0.8 \leq S t_{D_{w}} \leq 38$, keeping in mind that numerical dissipation and dispersion are expected to have substantial effect for $S t_{D_{w}} \geq 23$.

\subsection{Acoustic results}

\subsubsection{Evaluation of the solid surface integral and comparison with the literature}

We first turn to the evaluation of the solid FW-H computation, as it is less prone to numerical errors than the permeable one. $R_{\mathrm{obs}}$ is taken as $50 D_{w}$. The narrowband pressure spectra are presented in figure 16 and present several similarities with the CADWIE experimental [57] and numerical [33] studies. Indeed, the cavity induces a middle-frequency component mostly composed of a broad tone that corresponds to a cavity depth mode. This depth mode appears preferentially at the observers located in upsteam directions (P1 and P2), in coherence with the directivity of cavity noise. As for the global directivity, the OASPL polar
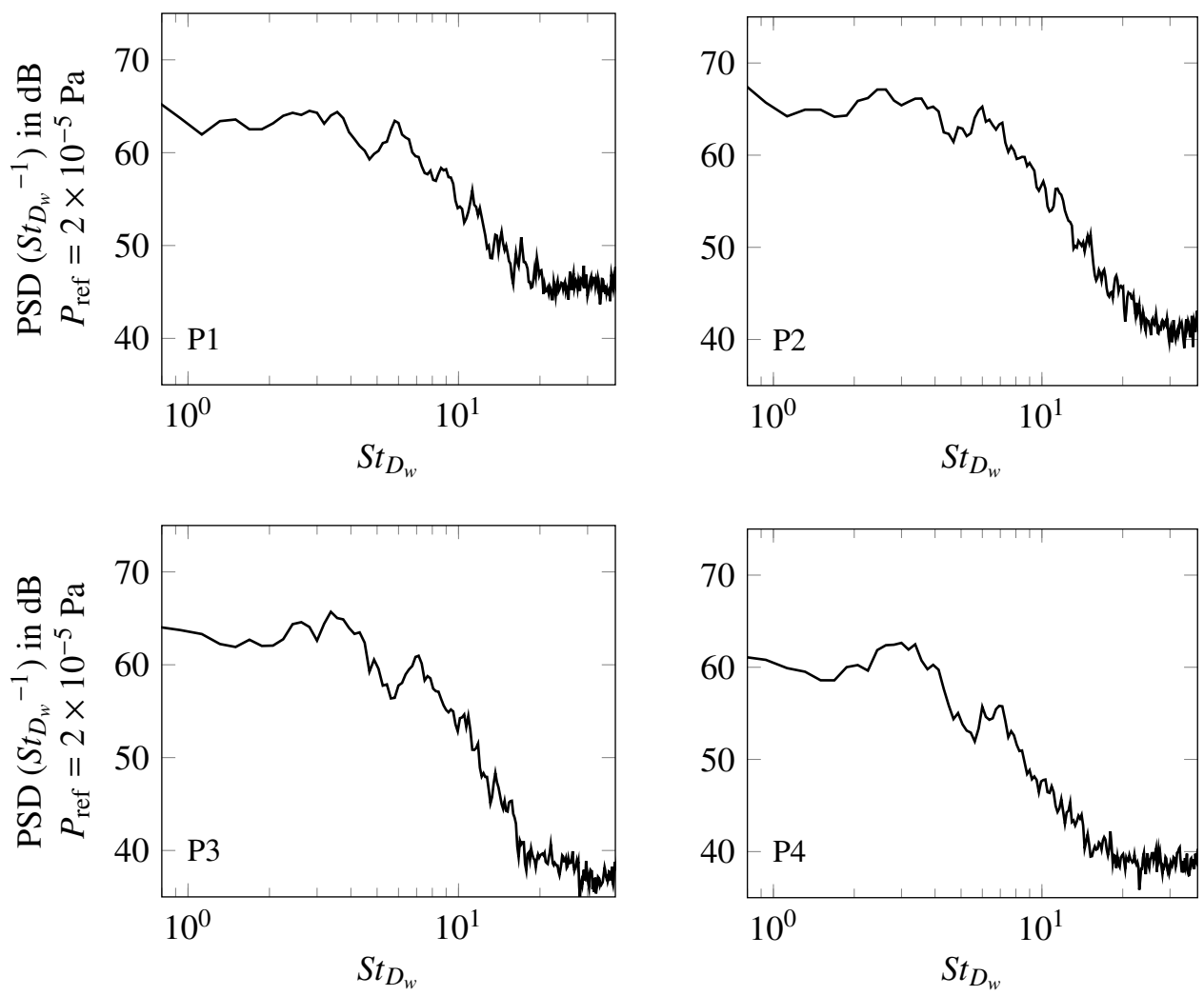

Figure 16: Far-field spectra obtained with the solid surface integral at different observation angles. Top-left: P1, top-right: P2, bottom-left: P3, bottom-right: P4 (see fig. 14.-left).

plot in the $y O z$ plane, presented in figure 17 -left, shows the same dipole shape as observed on the CADWIE wheel: a noise excess of about $4 \mathrm{~dB}$ is observed on the cavity side with respect to the external side. In the $x O z$ plane, the directivity plot clearly shows how the cavity is responsible for most of the acoustic energy, 400 preferentially in the upstream direction. 

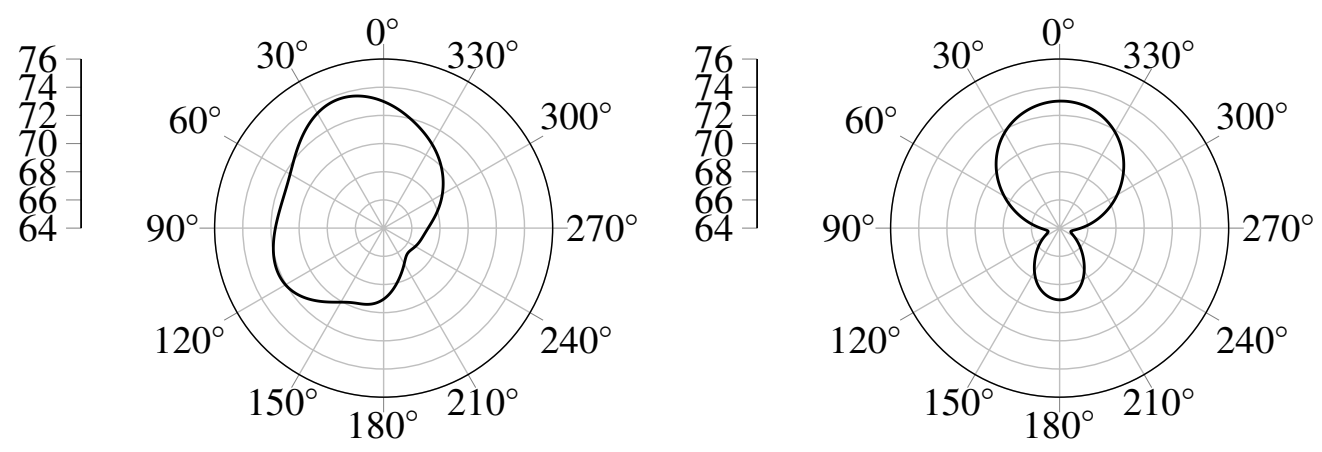

Figure 17: OASPL (dB) for the solid FW-H computation. Left: $x O z$ plane, right: $y O z$ plane.

\subsubsection{Permeable surface: effect of the near-field terms}

The observer distance $R_{\mathrm{obs}}$ is measured from the wheel center. Recalling that the permeable surface extends to $x / D_{w}=3.8$ in the streamwise direction and that our FW-H utility does not neglect any near-field terms, the pressure PSD at the four listener positions is presented in figure 18, using the finest permeable surface $\left(D_{w} / \Delta_{\Sigma}=60\right)$, according to Eq. 11 . The observer distance $R_{\mathrm{obs}}$ is varied by considering values of $5,10,50$, and $100 D_{w}$, and all the spectra are reduced assuming an inverse-distance decay, the reference spectrum corresponding to $R_{\mathrm{obs}, \text { ref }}=50 D_{w}$.

As expected, $\mathrm{P} 4$ presents a significant sensitivity to the observer distance, as shown in figure 18 -bottomright, the near-field terms of order $O\left(1 / d^{2}\right)$ and $O\left(1 / d^{3}\right)$ in Eq. 12 being still relatively important with respect to the $O(1 / d)$ terms at such distance. It is also interesting to note that at P1, the high-frequency content is dependent on the observer distance as well. The corresponding excess noise cannot be considered "spurious" a priori: it also reflects near-field contributions but in order to provide a meaningful comparison with the solid formulation (less extended integration surface), we wish to retain a value for $R_{\text {obs }}$ that does not present near-field effects in the permeable results. As the results stop varying from 50 to $100 D_{w}$, in what follows, all the computations with the permeable surface will be performed for observers located at a distance of $50 D_{w}$.

\subsubsection{Permeable surface: influence of the surface elements size}

As mentioned by Casalino et al. [58], the FW-H surface should ideally be composed of the CFD grid points. This would allow the best resolution, while avoiding possible interpolation errors between the CFD and the FW-H grids. In the present case, the storage cost would have been prohibitive, so the three surface resolutions described in section 4.1 have been compared. To obtain a satisfying numerical estimation of the surface integral, the surface elements must be small enough so they can be considered punctual. In the FW-H framework, the surface integral represents the sum of a distribution of equivalent sources, and the retarded-time differences must also be negligible on an individual surface element to ensure correct phase compensations. The results for the three resolutions are plotted in figure 19. The results obtained with the coarse surface exhibit much higher levels in every direction than its medium and fine counterparts, which might be due to unresolved phase compensations in the coarse case. According to figure 12, the wavelength of the significant eddies in the wake, expressed as a function of their phase speed and frequency as $\lambda^{*}=v_{\varphi} / f$, are found at $S t=1$, yielding $\lambda^{*}=v_{\varphi} D_{w} / U_{\infty}$. Then, by estimating the phase speed of these most significant eddies as $v_{\varphi}=U_{\infty} / 2$, we find that a certain number of points per eddies is needed in order not to contaminate the far-field. The presented dataset would suggest that in the present case, a spatial resolution of 15 points per eddies would be coherent with the result of figure 19 . A more systematic study 

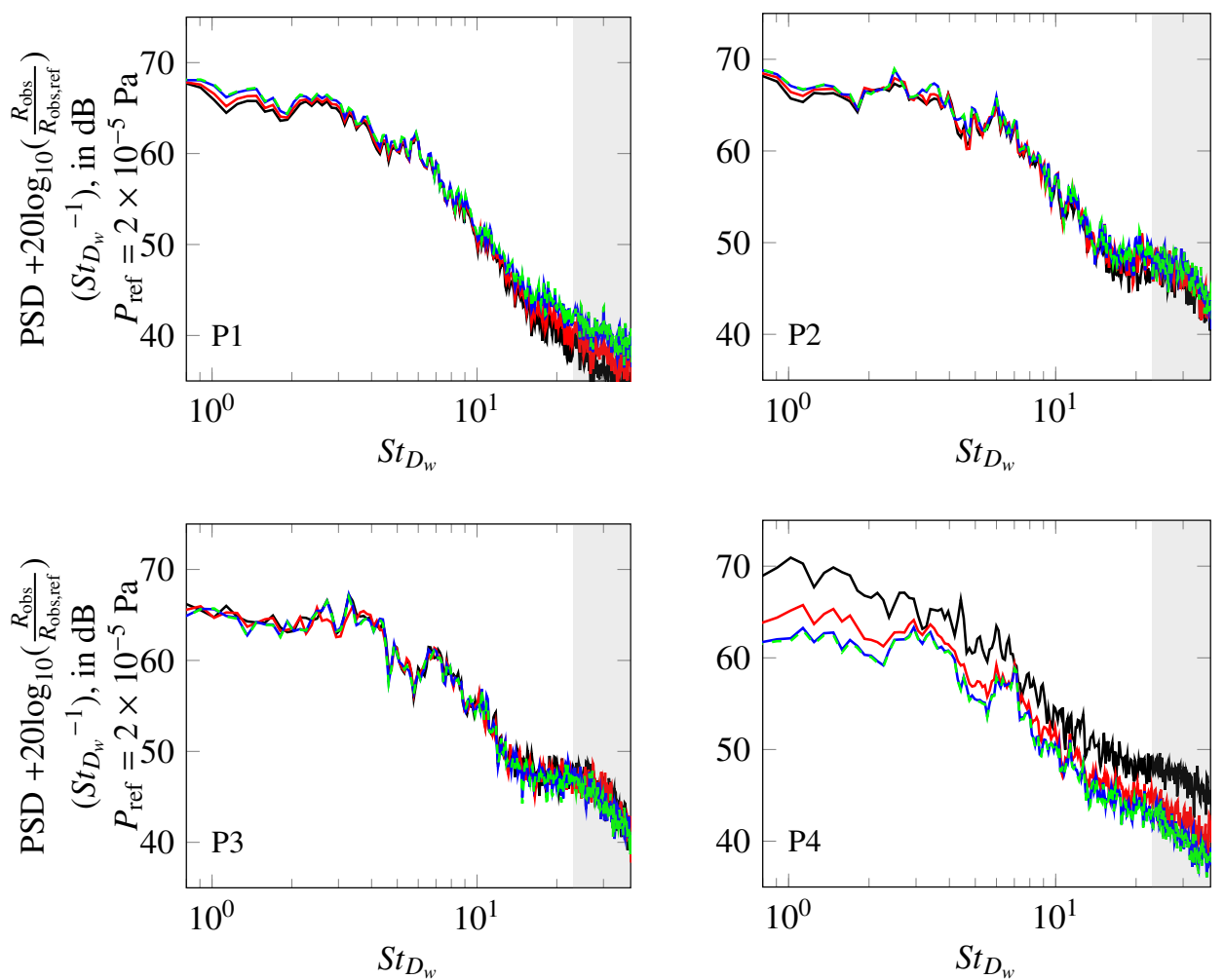

Figure 18: Far-field spectra obtained with the fine permeable surface $\left(\Delta_{\Sigma}=D_{w} / 60\right)$ at different observation angles, varying the $R_{\mathrm{obs}}$ value. (一): $R_{\mathrm{obs}}=5 D_{w},(-): R_{\mathrm{obs}}=10 D_{w},(-): R_{\mathrm{obs}, \text { ref }}=50 D_{w},(-): R_{\mathrm{obs}}=100 D_{w}$. Top-left: P1, top-right: P2, bottom-left: P3, bottom-right: P4 (see fig. 14.left). Gray area: CFD cutoff.

is considered in future works in order to complement this qualitative reasoning. Finally, although some differences still appear between the medium and the fine surfaces, especially at P1 and P4, refining the surface would have been too costly, so the fine surface will be retained for the analysis. While convergence of the surface elements size is not completely achieved at P1 and P4, it must be noted that P2 and P3 should be spared from this source of error.

\subsubsection{Effects related to the truncation of the source domain}

Another source of imperfection in FW-H computations is related to the fact that, in practice, the integration surface cannot possibly contain all of the turbulent volume. This results in turbulence eventually crossing the surface and the generation of spurious noise. Although the mathematical framework of the FW-H equation imposes a closed control surface, opening the outflow surface in order to overcome the difficulty induced by the wake turbulent eddies crossing the surface has become a common solution. The effect of opening the surface is presented in figure 20, where solid lines correspond, for a given surface discretisation, to the closed surface, and the dashed lines correspond to the corresponding open surface. It appears that when the surface is open, the same noise levels are obtained irrespective of the FWH surface resolution, showing that the effect of this parameter could be actually very localized on the downstream section of the integration surface, where an accurate description of the phase compensations appears to be critical.

When comparing to the results obtained with a closed downstream end, it also appears that opening 

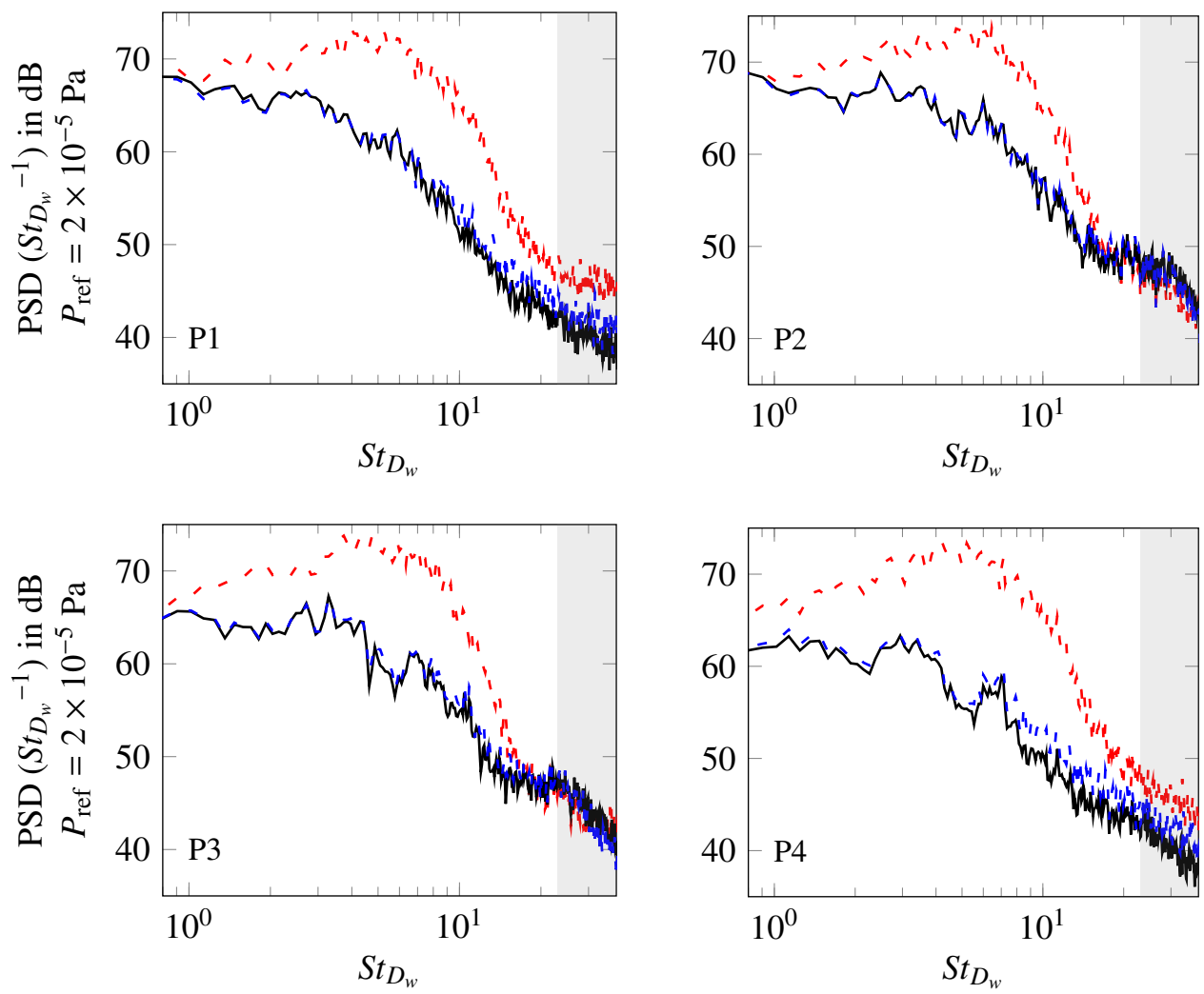

Figure 19: Far-field spectra obtained with the permeable surface integral at different observation angles, varying the $\Delta_{\Sigma}$ FW-H grid resolution. (- ): $\Delta_{\Sigma}=D_{w} / 15,(--): \Delta_{\Sigma}=D_{w} / 30,(-): \Delta_{\Sigma}=D_{w} / 60$. Top-left: P1, top-right: P2, bottom-left: P3, bottom-right: P4 (see fig. 14.left). Gray area: CFD cutoff.

the surface overall increases the very low frequencies and decreases the sound levels of about $10 \mathrm{~dB}$ in the highest frequency range, especially for P1 and P4 (see figures 20-top-left and bottom-right). Strictly speaking, nothing proves that the "closed" result is more accurate than the "open" one. However, if we compare the levels obtained with the solid formulation at P1 and P4, we can see that the closed surface is quantitatively way closer than the open one, showing that the "opening error" would be greater than the "quadrupole error" at these angles.

A better way to cope with the problem of turbulence leaving the surface is instead to directly approximate the volumetric contribution by additional surface terms that represent the flux of volume sources across the surface. Such terms have been developed at ONERA by Rahier et al. [39] from the original FW-H equation and have proven to greatly reduce the spurious noise while keeping the control surface closed in the case of turbulent jets. The results obtained with the fine surface and such correction are presented in figure 21 . Those obtained with the open control surface are also shown for comparison of both effects.

The examination of the spectra at P2 and P3 indicates that the influence of the corrective terms is limited to the low frequency range, with a spurious noise reduction of a few $\mathrm{dB}$. At these angles, except for the very lowest frequencies, opening the surface is almost equivalent to using the correction. On the other hand, at points $\mathrm{P} 1$, and especially $\mathrm{P} 4$, that are more sensitive to the downstream surface contribution, large differences are seen in the noise levels obtained with the closed and corrected surface on one hand, and the open surface on the other hand. In the low frequency range, the corrective terms decrease the levels 

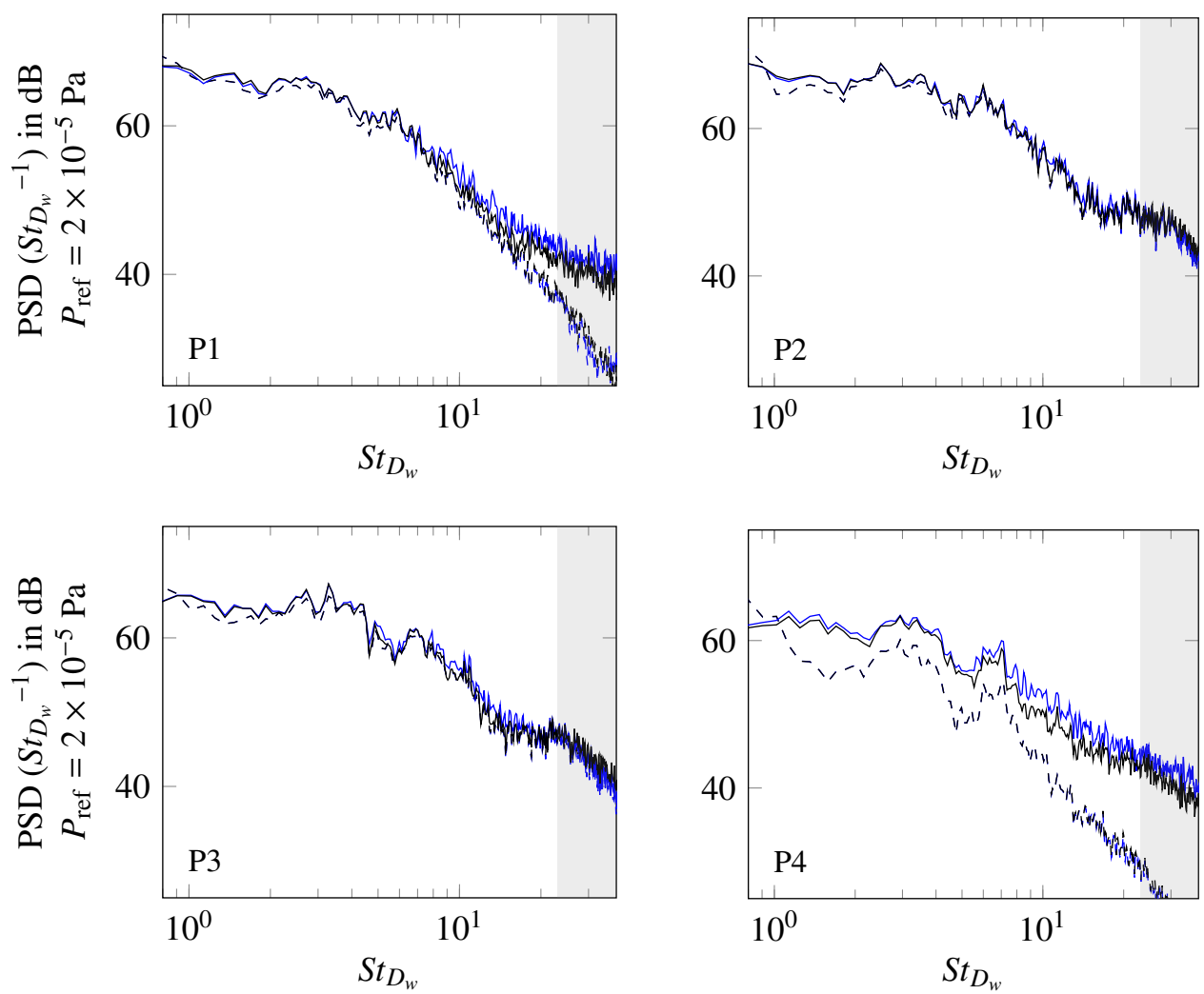

Figure 20: Far-field spectra obtained with the permeable surface integral at different observation angles. Combined effects of varying the $\Delta_{\Sigma}$ FW-H grid resolution and opening the surface. (-): $\Delta_{\Sigma}=D_{w} / 30,(-): \Delta_{\Sigma}=D_{w} / 60$. Solid lines: surface closed. Dashed lines: surface open. Top-left: P1, top-right: P2, bottom-left: P3, bottom-right: P4 (see fig. 14.left). Gray area: CFD cutoff.

by about $4 \mathrm{~dB}$, and avoid the seemingly unphysical decrease of the high frequencies observed with the open 470 surface.

The corrective terms influence seems thus restricted to the low frequencies, and therefore must have a substantial effect on the computed OASPL. Figure 22 shows the polar plot of the OASPL in the $x O z$ plane. The "corrected" directivity appears somehow more regular, and presents a shape that looks closer to what was obtained with the solid formulation. Therefore, our conclusion is that the use of corrective terms seems preferable than opening the surface as it allows to suppress the low frequency spurious noise, while preventing the unphysical drop of the high frequencies when the downstream end is ignored. Comparing surface averaging to these results could also be interesting, but has not been possible in the present case, as only one outflow surface was recorded during the simulation.

\subsubsection{Comparison between solid and permeable results}

As suggested by Casper et al. [59], differences in far-field radiation between the permeable and the solid FW-H control surface either suggest that there is a non-negligible volumetric contribution, or that the input flow simulation is suspect. In keeping with this idea, the previous paragraphs have been devoted to a careful examination of the points that could possibly go wrong in the computation of the FW-H integral on the permeable control surface. As a reminder, it has been checked that the flow was almost uniform on the outflow surface, with a local deficit of only $10 \%$ with respect to the upstream velocity. The volume mesh 

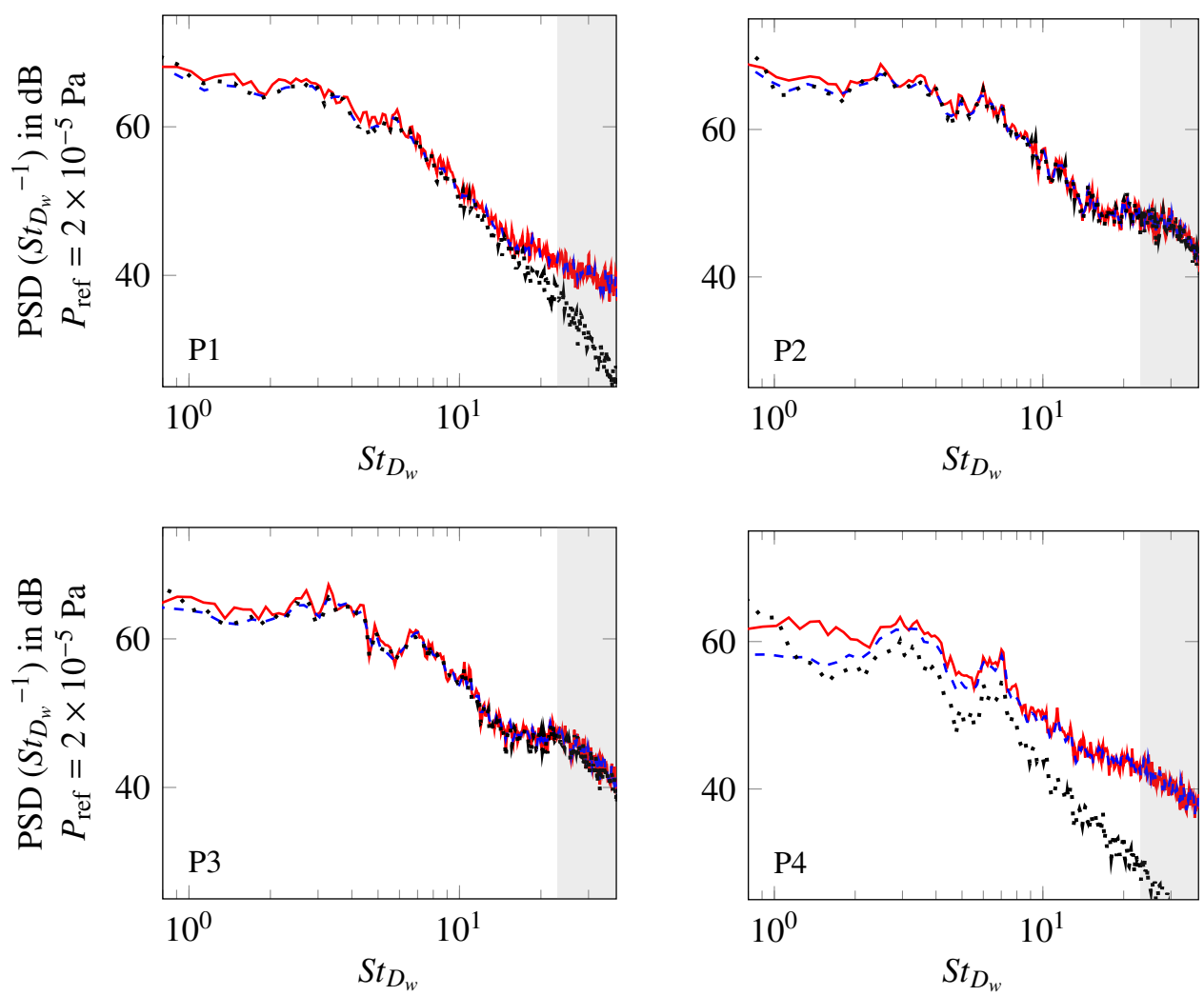

Figure 21: Far-field spectra obtained with the fine permeable surface $\left(\Delta_{\Sigma}=D_{w} / 60\right)$ at different observation angles. Assessment of the end-cap treatment. (-): closed surface with no correction, (- -): closed surface with correction, $(\cdots)$ : open surface. Top-left: P1, top-right: P2, bottom-left: P3, bottom-right: P4 (see fig. 14.left). Gray area: CFD cutoff.

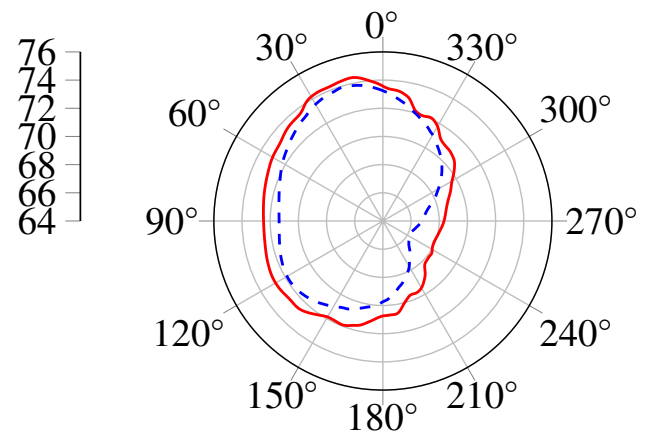

Figure 22: OASPL (dB) for the closed fine permeable surface $\left(\Delta_{\Sigma}=D_{w} / 60\right)$ in the $x O z$ plane. (-): without correction, (- -): with correction.

has been designed in order to accurately propagate the sound waves to the permeable surface up to the noncompact frequency range. The duration of the time signals, the near-field terms influence, the resolution of the surface integral, as well as the Lighthill stress tensor flux have been assessed in order to ensure results as unbiased as possible. It has also been checked that the acoustic computation was consistent with the theory in the sense that the pressure field vanishes inside the surface (see Appendix A).

All these points giving confidence in the permeable computation, we present the comparison of the 
far-field acoustic pressure power spectral density obtained with the two formulations in figure 23 . The $D_{w} / \Delta_{\Sigma}=60$ surface is used, with an observer distance $R_{\mathrm{obs}}=50 D_{w}$. The downstream end is kept closed with the use of the corrective terms.
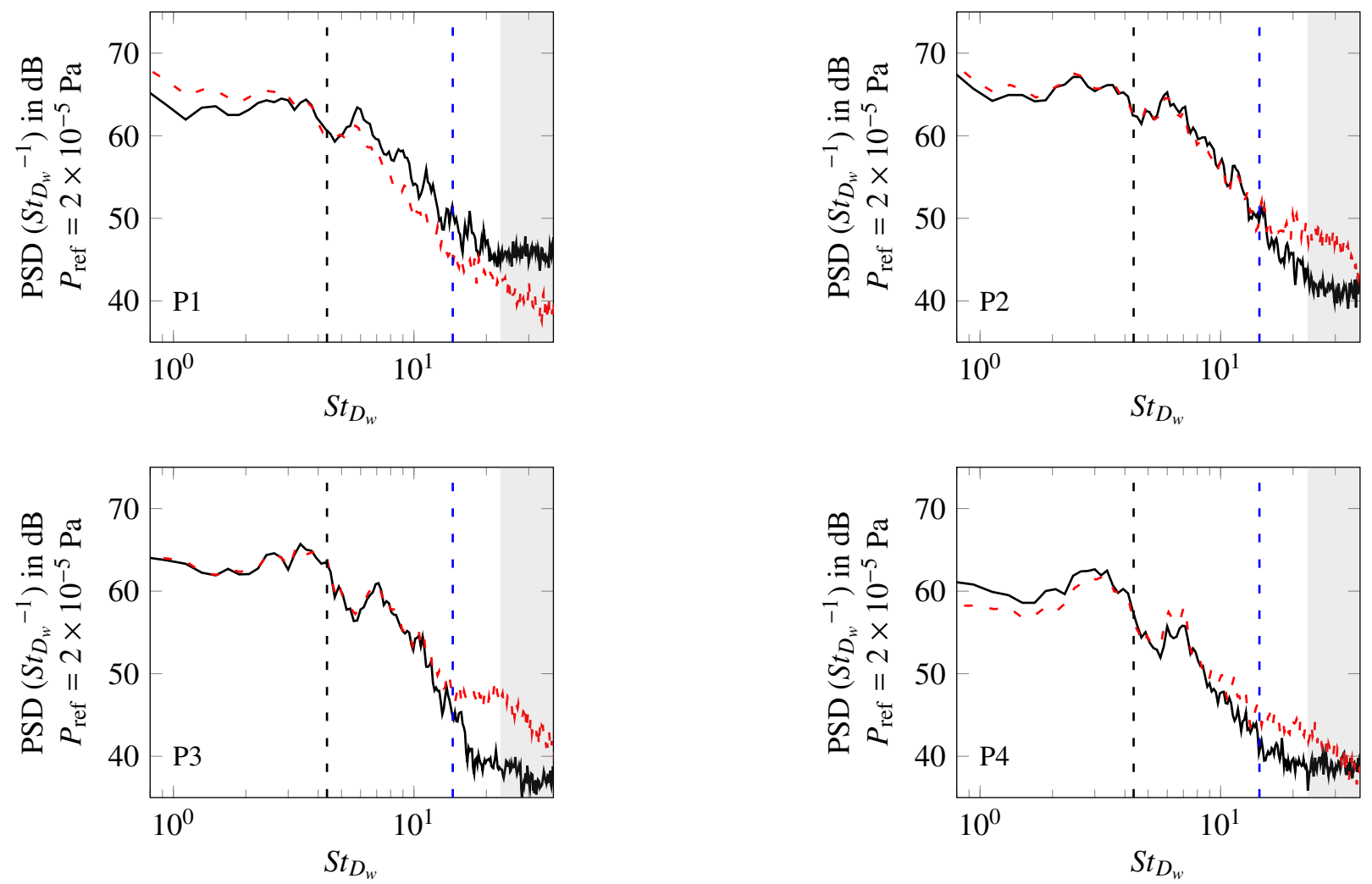

Figure 23: Comparison of the far-field pressure PSD obtained with both FW-H approaches. (-) : solid formulation, (- -) : permeable formulation. (- -):MSt $t_{D_{w}}=1,(--): M S t_{H_{w}}=1$. Gray area: CFD cutoff.

Using the wheel diameter as the reference length for dissociating the compact and non-compact frequency ranges yields a critical value $S t_{D_{w}}=1 / M=4.35$, shown by a dashed black line in fig. 23. The use of the wheel width $H_{w}$ yields $S t_{D_{w}}=14.5$ and is shown with a blue dashed line.

The analysis of grazing angles is more delicate so let us first proceed to the examination of the spectra obtained at points P2 and P3. At these two points, except a slight increase (of the order of $1 \mathrm{~dB}$ ) for $0.8 \leq S t_{D_{w}} \leq 2$ at P2, both formulations give identical noise levels within a fraction of dB when $S t_{D_{w}} \leq 11.5$. Numerically, such a good agreement between both formulations rewards a posteriori the computational effort invested on the wake mesh, as the quadrupoles inside the surface, as well as their propagation up to the FW-H surface appear very well described. This should be emphasized as an agreement between solid and permeable computations has not always been so clearly obtained on more complex landing gear flows. Recalling that the effect of corrective terms was mainly concentrated in the low frequency range also retrospectively confirms their relevance as it allows permeable results to match the solid ones in the compact frequency interval, as predicted by theory. When $S t_{D_{w}} \geq 11.5$, important discrepancies of up to $10 \mathrm{~dB}$ are found between both formulations. Such differences were erroneously attributed by the present authors to spurious noise in [35], a conclusion that seems invalidated by the present results, which show that the effect of turbulence leaving the surface only affects the low frequency range, and that these differences remain 
even when near-field terms are inefficient. The fact that both formulations predictions diverge when $\lambda=H_{w}$ supports the theory as it is consistent with the compact source argument, and prompts us to consider that the observed discrepancies are indeed physical, and not associated with the "end-cap problem".

Observers at grazing angles, such as P1 and P4, were more difficult to predict. In both cases, the spectra obtained with solid and permeable computations seem to present the same global shape. At P1, the levels obtained with the permeable approach are greater at low frequencies with respect to the solid formulation, while the opposite is observed at high frequencies. The mirror trend is observed at P4. At both angles, solid and permeable approaches yield the same noise levels around $M S t_{D_{w}}=1$, but this might be fortuitous. We would attribute this phenomenon to mean flow effects, not taken into account by the solid formulation, but this point would clearly deserve more work.

The OASPL is plotted in figure 24. Taking into account the quadrupoles with the permeable surface results in a slight increase of noise levels on the cavity side, but it is the external side that sees the most significant increase. The inclusion of quadrupoles results in an increase of about $3 \mathrm{~dB}$ in the directions of weaker radiation in the plane $y \mathrm{Oz}$. It also clearly appears that OASPL plots "hide" the differences highlighted in the narrowband spectra. This is even more striking at listeners $\mathrm{P} 1$ and P4, that present equal OASPL value, while the narrowband spectra differ considerably at these angles. Here, it is worth pointing out once again that OASPL are probably not the best representation to address the dipole/quadrupole question.
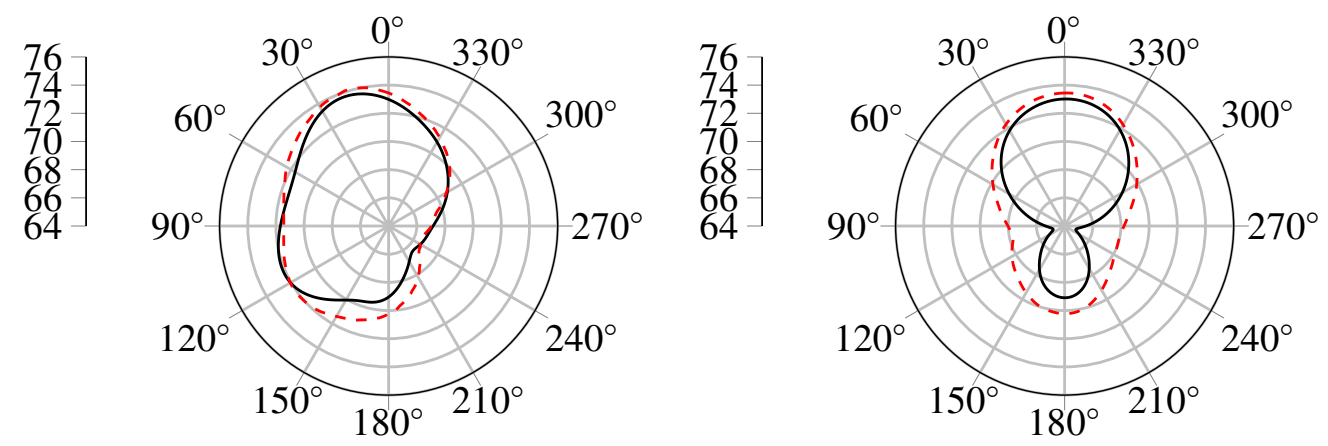

Figure 24: OASPL (dB) for the FW-H computations with the (-): solid approach, and (- -) permeable approach. Left: $x O z$ plane, right: $y O z$ plane.

\subsubsection{Comparison between FW-H results and Direct Noise Computation}

Perhaps the most reliable way to investigate the remaining differences between the solid and permeable formulation results obtained in figure 23 would be to compute the volume integral enclosed by the permeable surface. The sum of the solid surface dipoles with this volume integration should in theory be strictly equal to the results obtained with the permeable formulation. The required storage cost is however not affordable in our case, so a more tractable way of investigating these differences, especially in the high-frequency range (e.g. fig. 23-bottom-left), is to compare the results obtained with the solid formulation to the noise computed with the compressible CFD solver, ie to use Direct Noise Computation (DNC) as a reference result. This has been done by Zhang, Moreau \& Sanjosé [16] on the compressible wall-resolved LES of a circular cylinder with long spanwise length, and also by Spalart et al. [60] on various test cases using DDES. The near-field pressure probe considered in figure 13 has been retained for this purpose. We recall that, thanks to the mesh refinement inside the permeable surface that follows the 20 points per wavelength criterion, dissipation and dispersion errors up to this probe are expected to be negligible. In order to cope 


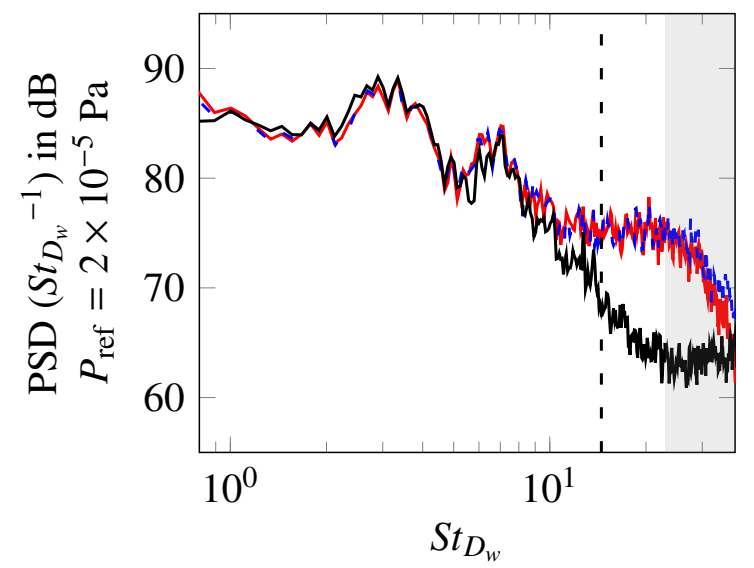

Figure 25: Near-field pressure PSD obtained with (- -): permeable approach, (一): solid approach, and (—): Direct Noise Computation $(\mathrm{DNC})$ at $\left(x / D_{w}, y / D_{w}, z / D_{w}\right)=(2.7,0,1.2)$. Vertical dashed line: $M S t_{H_{w}}=1$. Gray area: CFD cutoff.

${ }_{\mathrm{a}}$ with the irregular behavior of the acoustic field at distances $r$ such as $r \leq \Delta_{\Sigma}$, as pointed out in Appendix A. the permeable computation has been made at a distance $\varepsilon \simeq 2 \Delta_{\Sigma}$ from the true near-field point that is exactly located on the surface. Considering the small distance $\varepsilon$, this is not expected to be a source of any kind of error. Figure 25 presents the power spectral density with the solid and permeable methods. The DNC spectrum is shown for comparison, and the $M S t_{H_{w}}$ critical frequency is shown in dashed black line. This result is reminiscent of those obtained by Spalart et al. ([60], Fig. 8) and Zhang et al. ([16], Fig. 18-a) and supports once again that the excess high-frequency noise provided by the permeable approach is not due to imperfections in the FW-H integration, as initially inferred in Ref. [35]. It is also clear that using solely wall-pressure perturbations, even from a compressible simulation in the present case, leads to important underestimation of the noise levels whenever the body considered becomes non-compact. Determining the critical frequency up to which the solid formulation is still relevant would be a valuable information in an industrial context, and might constitute a perspective for future work. The wheel diameter was at first the prospective candidate, but it finally appears that the wheel width is in the present case more accurate. Lastly, it should be noted that the most reliable interpretation of figure 25 is that noise levels predicted with the solid approach are inferior to those predicted by DNC for the highest $S t$ values. The fact that DNC and permeable computation give the same spectrum might be due to the fact that the permeable result is mostly influenced by the local FW-H surface elements. This last result could therefore be, at best, a further consistency check of our FW-H utility.

\section{Noise source localization using a frequency-domain deconvolution algorithm}

The localization of aeroacoustic sources by means of phased microphone arrays has become a common practice to investigate airframe noise sources. While it has mostly been used as an experimental technique, nothing in principle precludes its use with computational data, as illustrated recently for instance on supersonic jet noise [61, 62], or circular cylinder noise [63]. If the virtual microphone array is beyond the accurate range of the simulation, the far-field sound can be obtained via FW-H integration. This gives to the initial question of determining which FW-H formulation is the most exact a practical interest, outside of the fundamental question of the dipole/quadrupole relative importance in low Mach number flows. Noting that frequency-domain methods such as DAMAS [64] involve the cross-spectral matrix on the microphone 
array, the aim of this last section is to determine how the differences in the FW-H noise spectra are reflected 570 in such results.

\subsection{Setup}

After some tests, a plane, cross-shaped array of virtual microphones, inspired by that used in Bulté \& Redonnet [65] on the LAGOON configuration in the "C19 open-jet" conditions has been retained. It is composed of two orthogonal linear arrays of 50 microphones evenly distributed on a distance of $100 D_{w}$. 575 One is aligned with the flow direction, while the other is orthogonal to it, as sketched in figure 26.
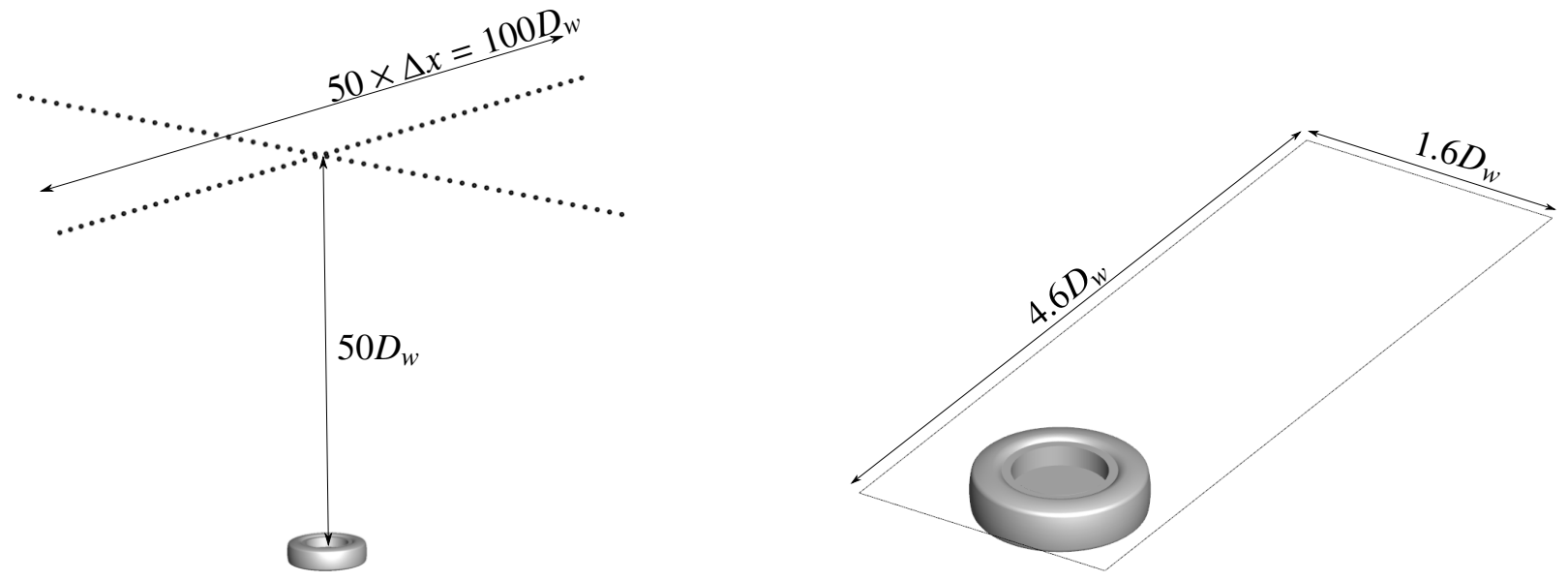

Figure 26: Numerical setup for the noise source localisation. Left: antenna (not to scale), right: scanning plane.

This array is located at a vertical distance of $50 D_{w}$ from the cavity bottom. With the angular convention adopted previously (see fig. 14-left), the angle $\varphi$ covered by the array lies between $-45^{\circ}$ and $+45^{\circ}$. Therefore, it is expected to be sensitive to the points raised at P2 and P3, but not to those observed at P1 and P4 that have not been fully understood yet.

The sources are sought in a scanning plane taken parallel to the array and grazing the cavity opening $\left(z / D_{w}=0.12\right)$. Its extent in the $x$ and $y$ directions corresponds to the fine grid zone of the CFD computation, which also coincides with the FW-H integration surface boundaries. It is divided in $46 \times 16$ square elements of size $c / D_{w}=0.1$.

\subsection{Acoustic maps}

The Welch estimator has been used to compute the cross-spectral matrix with $50 \%$ overlapping blocks to obtain a Strouhal resolution $\Delta S t_{D_{w}}=0.38$. A Hanning window is applied both in space and time so that side-effects are limited. The DAMAS iterative solver used features a Tikhonov regularization scheme in order to control the convergence of the solution at each frequency. Finally, for each frequency, contour plots are presented with a $10 \mathrm{~dB}$ dynamic range, starting from the maximum value.

In view of the observations made at observers P2 and P3 (cf. figure 23), the results are presented separately for $M S t_{H_{w}}<1$ in figure 27, and for higher Strouhal numbers in figure 28.

As shown earlier, the far-field spectra obtained with both FW-H formulations are almost identical at $S t_{H_{w}}$ values represented in figure 27, reflecting the fact that surface dipoles dominate in this frequency range. This similarity in far-field noise spectra is very logically reproduced in the computed noise maps. The main lobe decay is relatively slow for the lowest frequencies, but the spatial resolution of the array increases with frequency and for $M S t_{H_{w}} \geq 0.5$, the downstream corner of the cavity is clearly identified 


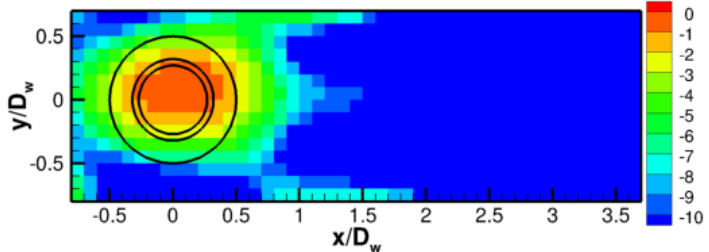

$M S t_{H_{w}}$

$=$

0.4
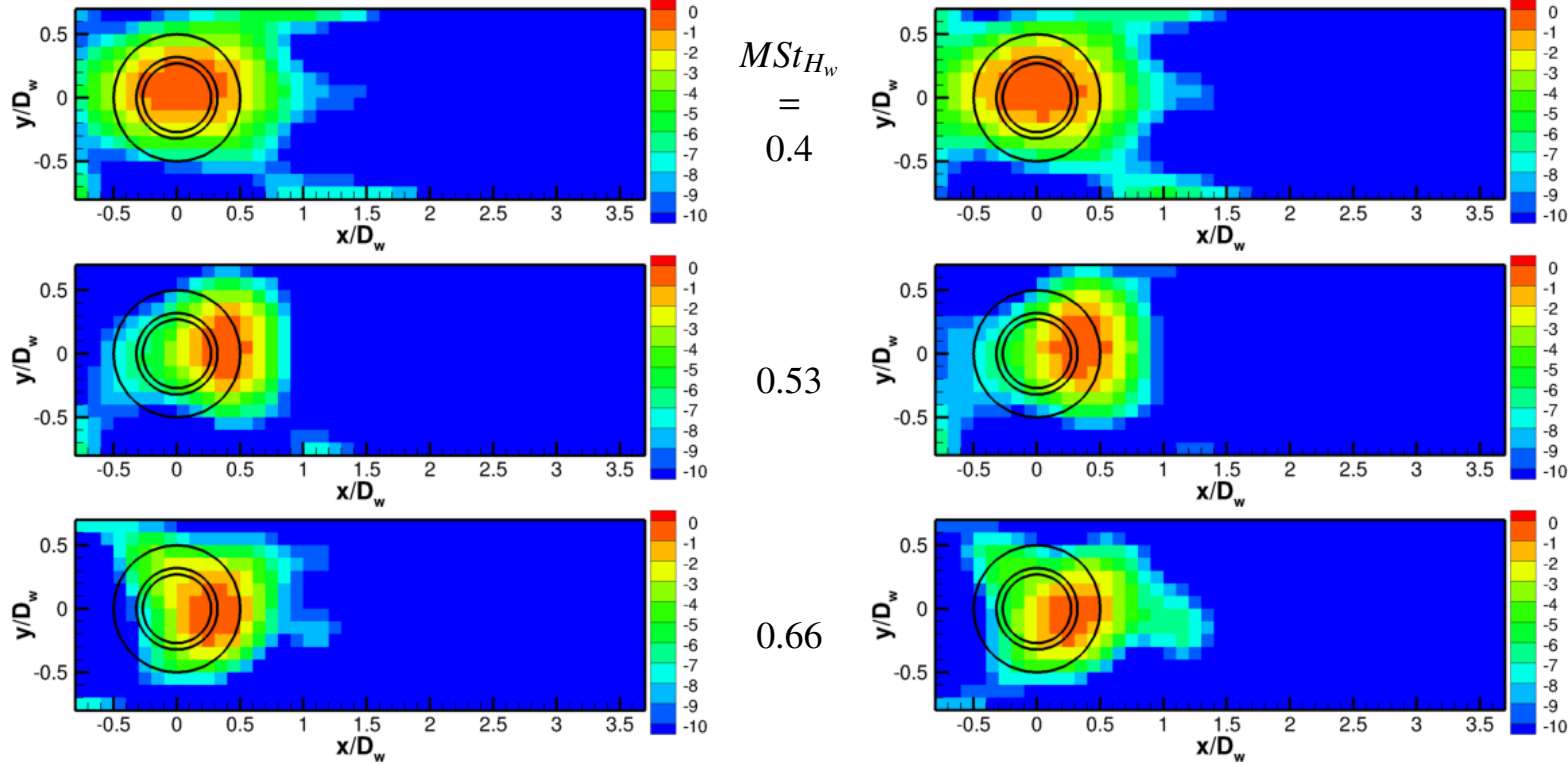

0.53

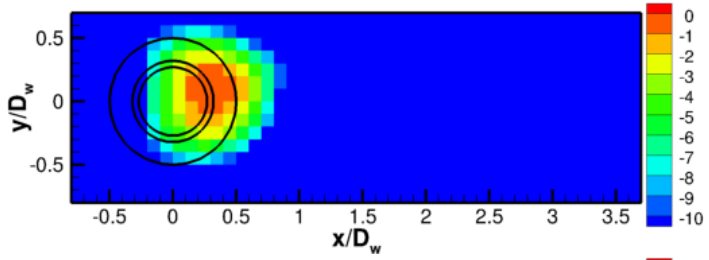

0.66

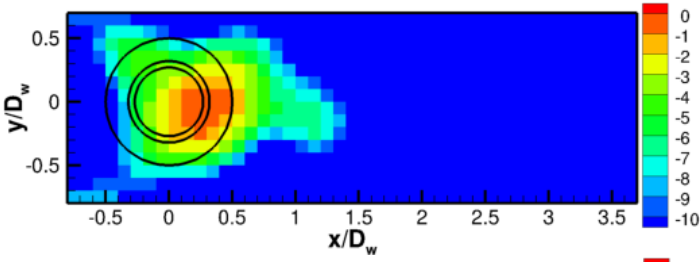

0.79
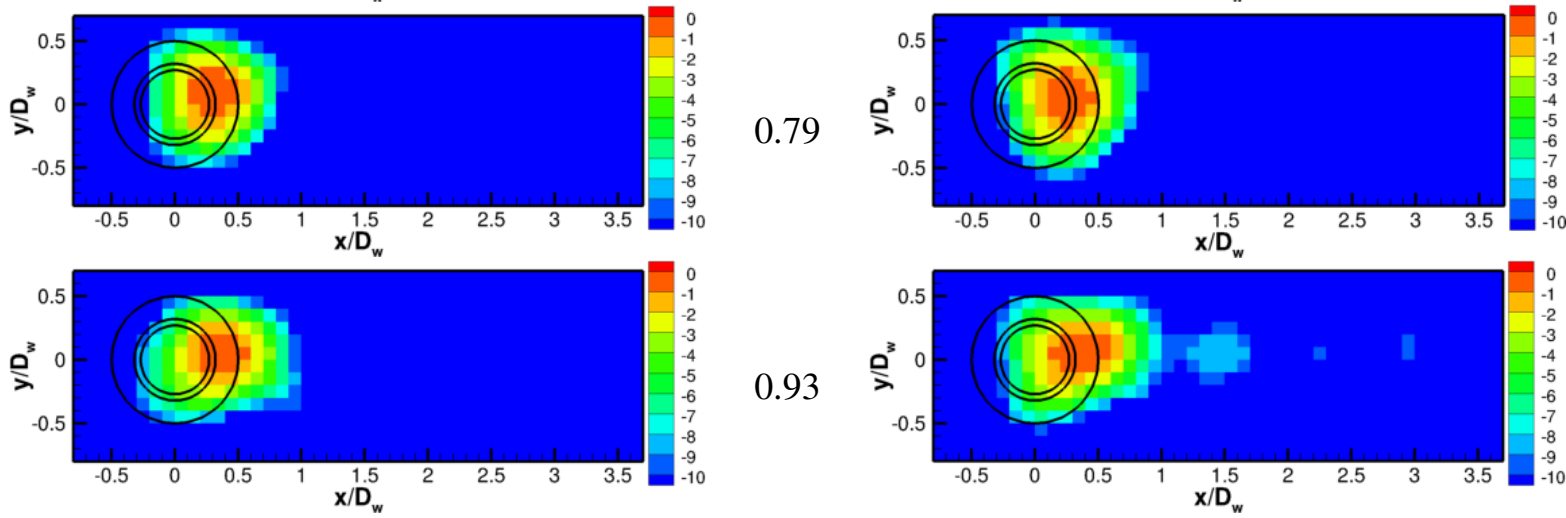

Solid

Permeable

Figure 27: Noise maps obtained in the $H_{w} / \lambda<1$ regime at $M S t_{H_{w}}$ values of (top-to-bottom): $0.4,0.53,0.66,0.79,0.93$. Input signals obtained with the (left): solid FW-H approach, and (right): permeable FW-H approach.

as the main source zone. Such a result, in accordance with what one would expect in this case, is also consistent with early observations made by Heller et al. [51] on shallow rectangular cavities, who showed that the main acoustic mechanism in this type of flow can be represented as an acoustic source located at the cavity downstream edge. Starting from $M S t_{H_{w}}=0.9$, as expected, the noise maps start to considerably differ, with the apparition of a more extended source zone in the downstream direction when the permeable method is used, with respect to the solid results.

This trend is confirmed by the examination of figure 28 . While the noise maps computed with the solid formulation still indicate the downstream cavity corner as the main source zone, those obtained with the permeable formulation show an extended source region that covers the cavity opening and the wheel wake, that could correspond to the regions of the flow where the "direct" contribution of the aerodynamic sources is emitted.

To summarize, a possible interpretation of theses results is that the dominant mechanism for $0.5 \leq$ $\left.M S t_{H_{w}} \leq 1\right)$ is the scattering of aerodynamic volume sources by the downstream edge of the cavity. At 


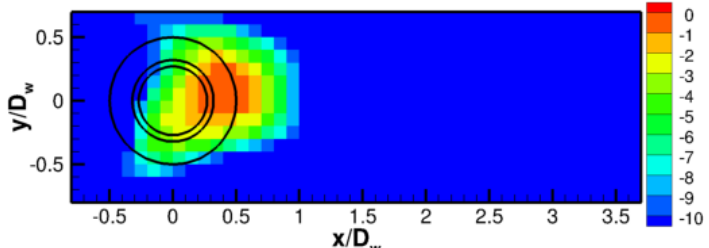

$M S t_{H_{w}}$

$=$

1.06
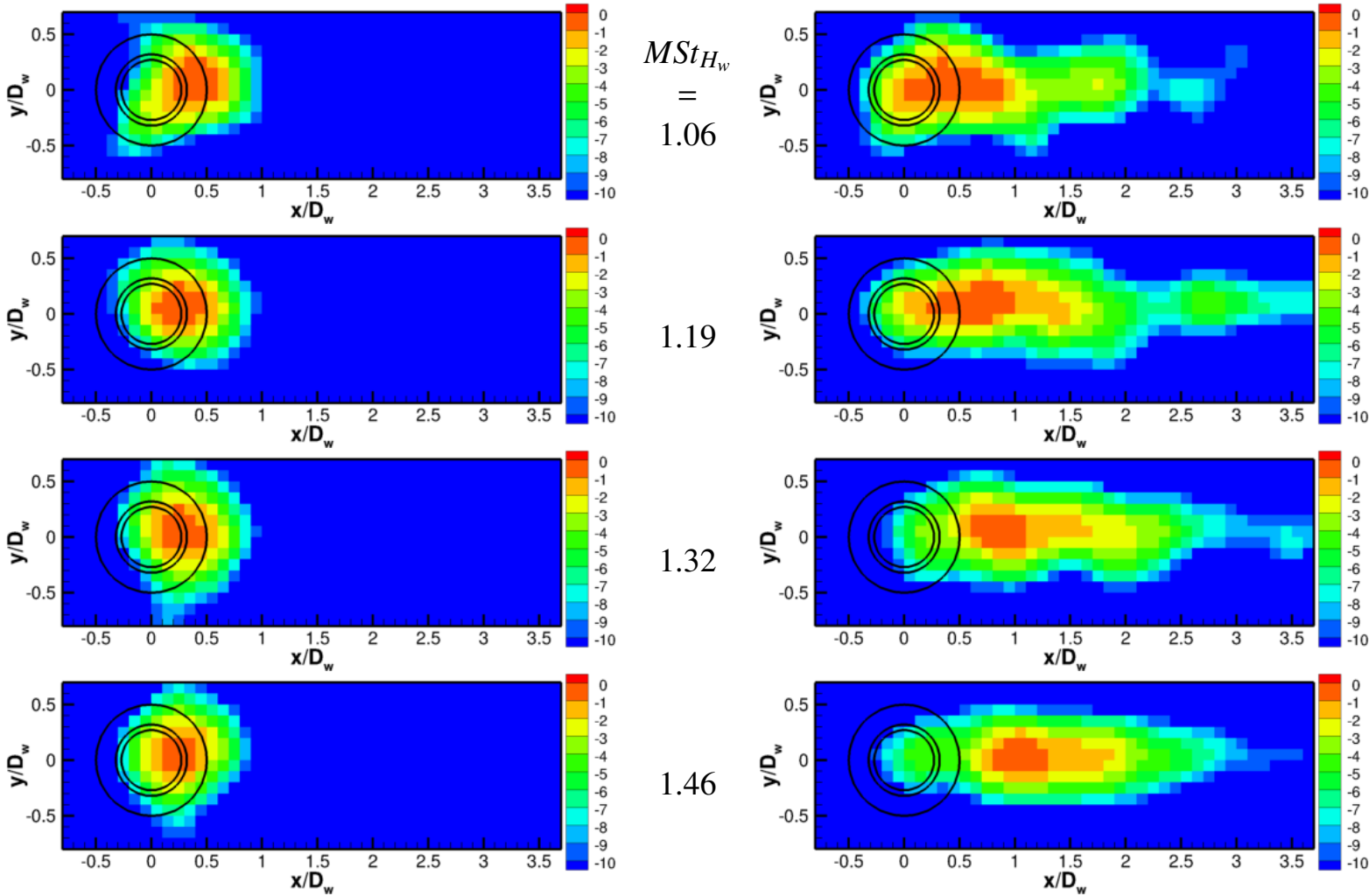

1.46
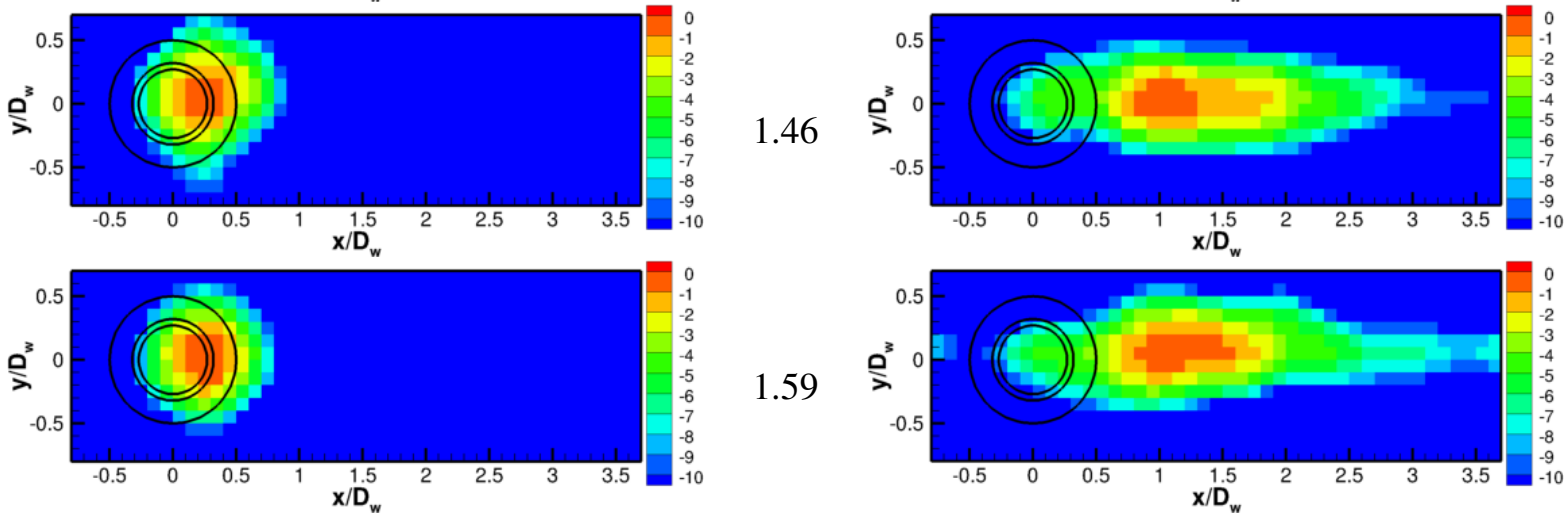

Solid

Permeable

Figure 28: Noise maps obtained in the $H_{w} / \lambda>1$ regime at $M S t_{H_{w}}$ values of (top-to-bottom): $1.06,1.19,1.32,1.46,1.59$. Input signals obtained with the (left): solid FW-H approach, and (right): permeable FW-H approach.

higher frequencies (or equivalently shorter wavelengths), the amplification of theses sources by scattering loses its importance relatively to the direct radiation of volume aerodynamic sources. Such an interpretation, if correct, would indicate that the interpretation of noise maps should be made carefully whenever the pressure signals are obtained with the FW-H analogy over non-compact bodies.

\section{Conclusions}

This work has been devoted to a comparison between the use of on- and off-body control surfaces for the FW-H equation in the case of an isolated landing gear wheel from the LAGOON configuration. The compressible turbulent flow has been solved with ONERA's code CEDRE by means of a ZDES approach, and acoustic computations have been performed using an extensively validated, time-domain, FW-H solver developed at ONERA. Careful attention has been paid to the propagation of acoustic perturbations to the permeable control surface, for sufficiently high frequencies with respect to the wheel dimensions, so the 
latter cannot be considered acoustically compact at all frequencies. The main idea was to challenge the compact source assumption that allows to rank the equivalent multipole terms on the basis of the usual $M^{6}$ and $M^{8}$ power laws.

An aerodynamic analysis and grid convergence study have been proposed and some flow features have been compared to similar geometries found in the literature, showing that the turbulent flow around the wheel is well described. The two counter-rotating vortex pairs downstream of the wheel are well captured by the simulation. The wake decay is also correctly resolved up to an almost self-similar state, showing that the immediate vicinity of the wheel wake is accurately described.

The well-known inherent difficulties related to the permeable surface method have led us to carefully examine some practical aspects related to the integral numerical evaluation, prior to any comparison with the solid surface method. The statistical convergence of the noise spectra has been proved. The surface near-field terms influence, and the control surface discretization have also been tackled. Removing the control surface end-cap has shown that, in the present case, the effect of the FW-H surface discretization is very localized on the downstream closure. On the other hand, opening the surface led to a dramatic decrease of about $10 \mathrm{~dB}$ for downstream observers in the highest frequency range, so this solution was not judged satisfying. Corrective terms that account for the Lighthill's tensor flux leaving the surface have been used and have decreased the noise levels in the low frequency range by a few $\mathrm{dB}$, while avoiding the unphysical behavior resulting from the surface opening. The numerical consistency of the acoustic computation has also been addressed by verifying that the computed pressure field inside the FW-H surface was close to zero, suggesting that the expected numerical uncertainty about our acoustic computation might be low.

Thereupon, a comparison between the solid and the permeable results has been made in the far-field, as well as in the near-field, DNC being used as a reference result in the latter case. In the far-field, observers located in directions nearly normal to the flow presented narrowband spectra identical with both formulations when the wheel can be considered compact based on the product $M \times S t$, the wheel width being taken as the reference length. Higher frequencies were substantially underestimated by the solid approach with respect to the permeable predictions in the far-field, but also to the "true sound of the simulation" obtained by Direct Noise Computation in the near-field. This interpretation is coherent with a previous theoretical argument provided by Davies [11] who stated that for large values of the $M \times S t$ product, the dominant radiation was of quadrupole type, while dipoles dominate whenever this product is small compared to unity.

Finally, an original illustration of this property has been proposed in the form of the noise sources localization by means of a frequency-domain source localization algorithm applied to the far-field signals computed with both the solid and permeable formulations. The scattering of aerodynamic sources by the downstream edge of the wheel circular cavity appears to be the dominant contributor to the noise when $M S t_{H_{w}}<1$. At higher $S t$ values, the "direct" field, taken into account by the permeable formulation, dominates and the noise maps reveal sources in the whole turbulent volume, that is over the cavity and in the wheel wake.

One of the reasons why the problem of knowing precisely whether quadrupoles play a significant role in low Mach number flows is not resolved yet is, in our opinion, due to the fact that it is probably a very case-dependent problem. For instance, as pointed out by a reviewer, the trailing-edge noise of an airfoil usually results from a strong scattering effect at the acute edge, but the solid FW-H method may suffice in many cases, although the airfoil chord is not compact at all. In CFD/CAA, the conclusions are also highly dependent on potential numerical inaccuracies. Even if the FW-H part is perfectly carried out, errors in the CFD part will necessarily be reflected in the noise results, and vice-versa. In that case, even DNC would not be the most relevant reference result. Both parts have been dealt with as carefully as possible in the present work, providing sound results with respect to theoretical reasoning. However, experimental 
measurements and/or further validation of our results are still needed to firmly confirm or contradict the proposed interpretations. Studying the influence of compressibility on solid results is also an avenue for future research. Indeed, CFL numbers well higher that unity are reached in the wall first prism layers, which led us to consider an implicit time scheme to meet affordable computational simulation time. A new run with a fully explicit setting could be of interest to evaluate the impact of these limited high CFL zones on the wall-pressure description accuracy for solid results. This could impact noise predictions at frequencies such that the wavelength is comparable to the prism layer height.

\section{Appendix A. Computation of the acoustic field inside the surface}

Equation 1 clearly highlights that the FW-H equation is valid everywhere in space, and amounts to resolving the field of the generalised variable $p^{\prime} H$, where $H$ is the Heaviside function, equal to zero inside the integration surface. One way to check the consistency of the FW-H computation is therefore to verify that the field inside the surface is zero, as done for instance by Spalart et al. [60]. In this respect, the pressure OASPL has been computed at 40 observer positions on a vertical line characterized by $x=y=0$, and $|z| D_{w} \mid \leq 1$. The OASPL profile thus obtained is presented in figure A.29. Inside the surface, delimited

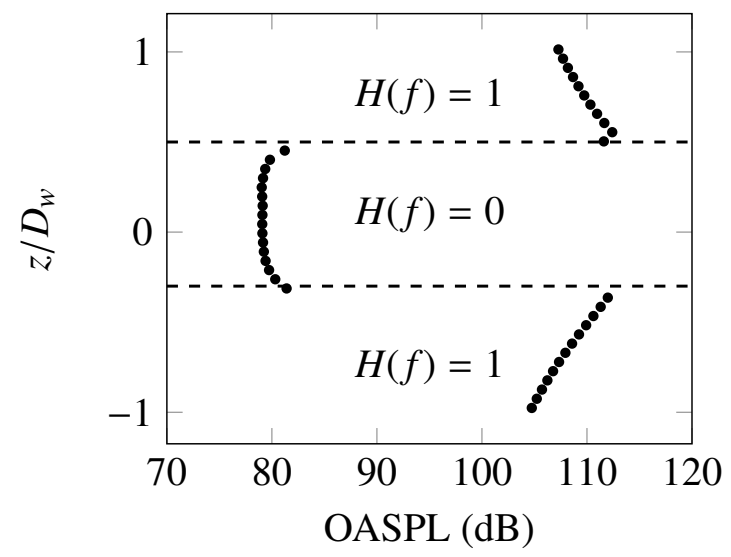

Figure A.29: OASPL profile obtained with the permeable surface computation in the near-field, along the vertical axis $x=y=0$ crossing the surface.

by the interval $-0.3 D_{w} \leq z / D_{w} \leq 0.5 / D_{w}$, the OASPL are about $30 \mathrm{~dB}$ inferior to those obtained outside the surface. The field is therefore not strictly speaking zero inside the surface, which could result from several factors. First, the mathematical surface is not of class $C^{1}$, and sharp angles could introduce a bias in the integral calculation. Second, even if special attention has been devoted to taking most of the volume sources into account, some of them have necessarily been left out. Finally, the aerodynamic field resulting from the $\mathrm{N}-\mathrm{S}$ solver is expected to comprise some error (presumably small but non zero). Additionally, it should be reminded that Spalart et al. [60] had in a similar problem a drop of about $26 \mathrm{~dB}$ inside the permeable surface, which they judged satisfactory.

A second remark can be made on the field calculated in the surface immediate vicinity. As we can notice, the latter presents an irregular, and probably non physical, behavior at the crossing of the $z=0.5 D_{w}$ and $z=-0.3 D_{w}$ planes. This is due to the fact that the observer distance must be of the order of the surface discretisation, so the surface appears continuous to the observer. This behavior is in our view purely numerical and restricted to the surface immediate vicinity. Consequently, we consider that it does not call 
our computation into question and that our FW-H gives a field that is consistent with the basic principles of the FW-H formalism.

\section{Acknowledgments}

This work is part of the first author's PhD thesis and has been funded by ONERA. It has considerably benefited from judicious comments by Dr. G. Rahier, as well as helpful critical comments from anonymous referees. The authors would also like to thank their colleagues from the DAAA/MAXE unit who provided the regularized DAMAS utility.

\section{References}

[1] M. R. Fink, Airframe Noise Prediction Method, Tech. rep., United Technologies Research Center East Hartford CT (1977).

[2] M. Smith, L. Chow, Prediction Method for Aerodynamic Noise from Aircraft Landing Gear, in: 4th AIAA/CEAS Aeroacoustics Conference, 1998, p. 2228.

[3] Y. Guo, A Statistical Model for Landing Gear Noise Prediction, Journal of Sound and Vibration 282 (1) (2005) 61-87.

[4] L. Hedges, A. Travin, P. Spalart, Detached-Eddy Simulations Over a Simplified Landing Gear, Journal of fluids engineering 124 (2) (2002) 413-423.

[5] F. J. Souliez, L. N. Long, P. J. Morris, A. Sharma, Landing Gear Aerodynamic Noise Prediction Using Unstructured Grids, International Journal of Aeroacoustics 1 (2) (2002) 115-135.

[6] D. Lockard, M. Khorrami, F. Li, High resolution calculation of a simplified landing gear, in: 10th AIAA/CEAS Aeroacoustics Conference, 2004, p. 2887.

[7] F. de la Puente Cerezo, L. Sanders, F. Vuillot, P. Druault, E. Manoha, Zonal Detached Eddy Simulation of a Simplified Nose Landing Gear for Flow and Noise Predictions Using an Unstructured Navier-Stokes Solver, Journal of Sound and Vibration 405 (2017) $86-111$.

[8] Q. Bouvy, B. Petot, T. Rougier, Review of Landing Gear Acoustic Research at Messier-Bugatti-Dowty, in: 22nd AIAA/CEAS Aeroacoustics Conference, 2016, p. 2770.

[9] J. F. Williams, D. L. Hawkings, Sound Generation by Turbulence and Surfaces in Arbitrary Motion, Philosophical Transactions of the Royal Society of London 264 (1151) (1969) 321-342.

[10] P. R. Spalart, On the Precise Implications of Acoustic Analogies for Aerodynamic Noise at Low Mach Numbers, J. Sound and Vib. 332 (11) (2013) 2808-2815.

[11] H. G. Davies, The Radiated Fields of Multipole Point Sources Near a Solid Spherical Surface, Journal of Fluid Mechanics 43 (3) (1970) 597-606.

[12] X. Gloerfelt, F. Pérot, C. Bailly, D. Juvé, Flow-Induced Cylinder Noise Formulated as a Diffraction Problem for Low Mach Numbers, Journal of Sound and Vibration 287 (1-2) (2005) 129-151.

[13] H. Fuchs, R. Armstrong, Turbulent Source Coherence and Helmholtz Number as Aerodynamic Noise Parameters, in: Structure and Mechanisms of Turbulence II, Springer, 1978, pp. 189-201.

[14] W. M. Dobrzynski, Airframe Noise - Landing Gear Noise, 2010.

[15] F. Pérot, J.-M. Auger, H. Giardi, C. Bailly, D. Juvé, Computation of the Noise Generated by Low Mach Number Flows Around a Cylinder and a Wall-Mounted Half Cylinder, in: 10th AIAA/CEAS Aeroacoustics Conference, 2004, p. 2859.

[16] C. Zhang, S. Moreau, M. Sanjosé, Turbulent Flow and Noise Sources on a Circular Cylinder in the Critical Regime, AIP Advances 9 (8) (2019) 085009.

[17] G. A. Bres, D. Freed, M. Wessels, S. Noelting, F. Pérot, Flow and Noise Predictions for the Tandem Cylinder Aeroacoustic Benchmark, Physics of Fluids 24 (3) (2012) 036101.

[18] B. Greschner, F. Thiele, M. C. Jacob, D. Casalino, Prediction of Sound Generated by a Rod-airfoil Configuration Using EASM DES and the Generalised Lighthill/FW-H Analogy, Computers \& fluids 37 (4) (2008) 402-413.

[19] J.-C. Giret, A. Sengissen, S. Moreau, M. Sanjosé, J.-C. Jouhaud, Noise Source Analysis of a Rod-Airfoil Configuration Using Unstructured Large-Eddy Simulation, AIAA Journal 53 (4) (2014) 1062-1077.

[20] M. C. Jacob, J. Boudet, D. Casalino, M. Michard, A Rod-Airfoil Experiment as a Benchmark for Broadband Noise Modeling, Theoretical and Computational Fluid Dynamics 19 (3) (2005) 171-196.

[21] W. R. Wolf, A. V. G. Cavalieri, B. Backes, E. Morsch-Flho, J. L. F. Azevedo, Sound and Sources of Sound in a Model 740 Problem with Wake Interaction, AIAA Journal 53 (9) (2015) 2588-2606.

[22] C. Yu, S. K. Lele, Volume Noise Sources in Turbulent Wake Interaction Problems: True Quadrupole Noise?, in: 20th AIAA/CEAS Aeroacoustics Conference, 2014, p. 3042. 
[23] P. R. Spalart, M. L. Shur, M. K. Strelets, A. K. Travin, Initial Noise Predictions for Rudimentary Landing Gear, J. Sound and Vib. 330 (17) (2011) 4180-4195.

[24] F. de la Puente, Aeroacoustic Simulations of Landing Gears with Unstructured Grids and a ZDES Turbulence Model, Ph.D. thesis, University Pierre et Marie Curie - Paris 6 (2017).

[25] J. Appelbaum, B. M. Duda, E. Fares, M. R. Khorrami, Airframe Noise Simulations of a Full-Scale Aircraft, in: 2018 AIAA/CEAS Aeroacoustics Conference, American Institute of Aeronautics and Astronautics, 2018.

[26] L. Sanders, E. Manoha, S. Ben Khelil, C. Francois, LAGOON: CFD/CAA Coupling for Landing Gear Noise and Comparison with Experimental Database, in: 17th AIAA/CEAS Aeroacoustics Conference (32nd AIAA Aeroacoustics Conference), 2011, p. 2822.

[27] F. De La Puente, L. Sanders, F. Vuillot, E. Manoha, Nose Landing Gear Flow and Noise Predictions on Unstructured Grid Using a Cell-Centered Navier-Stokes Code, in: 21st AIAA/CEAS Aeroacoustics Conference, 2015, pp. 1-14.

[28] N. S. Zawodny, Aeroacoustic Characterization of Scaled Canonical Nose Landing Gear Configurations, Ph.D. thesis, University of Florida (2013).

[29] S. Windiate, An experimental and numerical study of bluff body, Ph.D. thesis, University of Southampton (2014).

[30] J.-C. Giret, Simulations aux Grandes échelles des écoulements Instationnaires Turbulents Autour des Trains d'Atterrissage pour la Prédiction du Bruit Aérodynamique [large eddy simulation of unsteady turbulent flows for landing gear noise predictions], Ph.D. thesis, Université de Toulouse, pp. 172-179 [In English] (2014).

[31] D. Casalino, A. F. Ribeiro, E. Fares, Facing Rim Cavities Fluctuation Modes, Journal of Sound and Vibration 333 (13) (2014) 2812-2830.

[32] S. Spagnolo, Unsteady Aerodynamic Loads on Aircraft Landing Gear, Ph.D. thesis, University of Southampton (2016).

[33] M. Wang, High-Order Numerical Investigations into Landing Gear Wheel Noise, Ph.D. thesis, University of Southampton (2017).

[34] F. De La Puente, L. Sanders, F. Vuillot, P. Druault, Investigation on Landing Gear Shallow Round Cavity Flow Field and Noise Signature, in: 22nd AIAA/CEAS Aeroacoustics Conference, AIAA paper 2016-2774, 2016.

[35] A. Hajczak, L. Sanders, F. Vuillot, P. Druault, Investigation of the Ffowcs-Williams and Hawkings Analogy on an Isolated Landing Gear Wheel, in: 2018 AIAA/CEAS Aeroacoustics Conference, AIAA paper 2018-3301, 2018.

[36] P. Di Francescantonio, A New Boundary Integral Formulation for the Prediction of Sound Radiation, Journal of Sound and Vibration 202 (4) (1997) 491-509.

[37] D. Crighton, F. Leppington, On the Scattering of Aerodynamic Noise, Journal of Fluid Mechanics 46 (3) (1971) $577-597$.

[38] C. Schram, J. Anthoine, A. Hirschberg, Calculation of Sound Scattering Using Curle's Analogy for Non-Compact Bodies, in: 11th AIAA/CEAS Aeroacoustics Conference, AIAA paper 2005-2836, 2005.

[39] G. Rahier, M. Huet, J. Prieur, Additional Terms for the Use of Ffowcs Williams and Hawkings Surface Integrals in Turbulent Flows, Comp. \& Fluids 120 (2015) 158-172.

[40] P. Martínez-Lera, C. Schram, Correction Techniques for the Truncation of the Source Field in Acoustic Analogies, The Journal of the Acoustical Society of America 124 (6) (2008) 3421-3429.

[41] T. Ikeda, S. Enomoto, K. Yamamoto, K. Amemiya, Quadrupole Corrections for the Permeable-Surface Ffowcs WilliamsHawkings Equation, AIAA Journal (2017) 2307-2320.

[42] M. L. Shur, P. R. Spalart, M. K. Strelets, Noise Prediction for Increasingly Complex Jets. Part I: Methods and Tests, International journal of aeroacoustics 4 (3) (2005) 213-245.

[43] J. Prieur, G. Rahier, Aeroacoustic Integral Methods, Formulation and Efficient Numerical Implementation, Aerospace Science and Technology 5 (7) (2001) 457-468.

[44] E. Manoha, J. Bulté, B. Caruelle, LAGOON: an Experimental Database for the Validation of CFD/CAA Methods for Landing Gear Noise Prediction, in: 14th AIAA/CEAS Aeroacoustics Conference, AIAA paper 2008-2816, 2008.

[45] E. Manoha, J. Bulté, V. Ciobaca, B. Caruelle, LAGOON: Further Analysis of Aerodynamic Experiments and Early Aeroacoustics Results, in: 15th AIAA/CEAS Aeroacoustics Conference, AIAA paper 2009-3277, 2009.

[46] E. Manoha, B. Caruelle, Summary of the LAGOON Solutions from the Benchmark Problems for Airframe Noise Computations-III Workshop, in: 21st AIAA/CEAS Aeroacoustics Conference AIAA paper 2015-2846, 2015.

[47] M. M. Zdravkovich, A. J. Flaherty, M. G. Pahle, I. A. Skelhorne, Some Aerodynamic Aspects of Coin-Like Cylinders, Journal of Fluid Mechanics 360 (1998) 7384.

[48] J. Rossiter, Wind Tunnel Experiments on the Flow Over Rectangular Cavities at Subsonic and Transonic Speeds, Tech. rep., Ministry of Aviation; Royal Aircraft Establishment; RAE Farnborough (1964).

[49] O. Marsden, C. Bailly, C. Bogey, E. Jondeau, Investigation of Flow Features and Acoustic Radiation of a Round Cavity, 795 Journal of Sound and Vibration 331 (15) (2012) 3521-3543.

[50] O. Marsden, C. Bogey, C. Bailly, Investigation of Flow Features Around Shallow Round Cavities Subject to Subsonic Grazing Flow, Physics of Fluids 24 (12) (2012) 125107.

[51] H. H. Heller, D. Holmes, E. E. Covert, Flow-Induced Pressure Oscillations in Shallow Cavities, Journal of sound and Vibra- 
tion 18 (4) (1971) 545-553.

[52] A. Refloch, B. Courbet, A. Murrone, P. Villedieu, C. Laurent, P. Gilbank, J. Troyes, L. Tessé, G. Chaineray, J. Dargaud, E. Quémerais, F. Vuillot, CEDRE software, AerospaceLab (2) (2011).

[53] S. Deck, Recent Improvements in the Zonal Detached Eddy Simulation (ZDES) Formulation, Theoretical and Computational Fluid Dynamics 26 (6) (2012) 523-550.

[54] B. S. Lazos, Mean Flow Features Around the Inline Wheels of Four-Wheel Landing Gear, AIAA journal 40 (2) (2002) 805 193-198.

[55] B. Lazos, Surface Topology on the Wheels of a Generic Four-Wheel Landing Gear, AIAA journal 40 (12) (2002) $2402-2411$.

[56] B. S. Lazos, Reynolds Stresses Around the Wheels of a Simplified Four-Wheel Landing Gear, AIAA journal 42 (1) (2004) 196-198.

[57] X. Zhang, Z. Ma, M. Smith, M. R. Sanderson, Aerodynamic and acoustic measurements of a single landing gear wheel, in: 810 19th AIAA/CEAS Aeroacoustics Conference, 2013, p. 2160.

[58] D. Casalino, M. Jacob, M. Roger, Prediction of Rod-Airfoil Interaction Noise Using the Ffowcs-Williams-Hawkings Analogy, AIAA journal 41 (2) (2003) 182-191.

[59] J. Casper, D. Lockard, M. Khorrami, C. Streett, Investigation of Volumetric Sources in Airframe Noise Simulations, in: 10th AIAA/CEAS Aeroacoustics Conference, AIAA paper 2004-2805, 2004.

[60] P. R. Spalart, K. V. Belyaev, M. L. Shur, M. Kh Strelets, A. K. Travin, On the Differences in Noise Predictions Based on Solid and Permeable Surface Ffowcs Williams-Hawkings Integral Solutions, International Journal of Aeroacoustics 18 (6-7) (2019) 621-646.

[61] Y. Du, P. Morris, Numerical Investigation of the Noise Source Locations of Supersonic Jets Using the Beamformed Method, in: 50th AIAA Aerospace Sciences Meeting including the New Horizons Forum and Aerospace Exposition, 2012, p. 1169.

[62] P. Panickar, N. Sinha, N. Murray, Localization of Acoustic Sources in Shock-Containing Jet Flows Using Phased Array Measurements, in: 51st AIAA Aerospace Sciences Meeting including the New Horizons Forum and Aerospace Exposition, 2013, p. 613.

[63] Q. Zhao, Sound Source Localization of Flow Around Circular Cylinder by a Virtual Microphone Array Technique, AIP Advances 8 (5) (2018) 055130.

[64] T. F. Brooks, W. M. Humphreys, A Deconvolution Approach for the Mapping of Acoustic Sources (DAMAS) Determined from Phased Microphone Arrays, Journal of Sound and Vibration 294 (4-5) (2006) 856-879.

[65] J. Bulté, S. Redonnet, Landing Gear Noise Identification Using Phased Array with Experimental and Computational Data, AIAA Journal 55 (11) (2017) 3839-3850. 